\title{
Glassy Spin Dynamics in Geometrically Frustrated Buckled Colloidal Crystals
}

\author{
Di Zhou, ${ }^{1,2}$ Feng Wang, ${ }^{1}$ Bo Li, ${ }^{1}$ Xiaojie Lou, ${ }^{2,3}$ and Yilong Han ${ }^{1,4}$ \\ ${ }^{1}$ Department of Physics, Hong Kong University of Science and Technology, \\ Clear Water Bay, Hong Kong, China \\ ${ }^{2}$ Frontier Institute of Science and Technology, and State Key Laboratory for \\ Mechanical Behavior of Materials, Xi'an Jiaotong University, Xi'an 710049, China \\ ${ }^{3}$ MOE Key Laboratory for Nonequilibrium Synthesis and Modulation of Condensed Matter, \\ Xi'an Jiaotong University, Xi'an 710049, China \\ ${ }^{4}$ The HKUST Shenzhen Research Institute, Shenzhen 518057, China \\ (Received 10 September 2016; revised manuscript received 4 January 2017; published 25 May 2017)
}

\begin{abstract}
Geometrical frustration arises when the lattice geometry prevents local interaction energies from minimizing simultaneously. Whether and how geometrically frustrated spins or charges in clean crystals exhibit glassy dynamics remain elusive due to the lack of measurements on microscopic dynamics. Here, we employ buckled monolayer colloidal crystals to mimic frustrated antiferromagnetic Ising spins on triangular lattices and measure single-spin dynamics using video microscopy. Both attractive and repulsive colloidal crystals buckled into zigzag stripes with glassy dynamics at low effective temperatures in experiment and simulation. The simple local spin configurations enable uncovering correlations among structure, dynamics, and soft vibrational modes. Machine learning analysis further reveals facilitated dynamics to be an important mechanism of structural relaxation. Moreover, our simulation reveals a similar structure and dynamics in lattice Coulomb liquids. Hence, spin-lattice coupling and long-range interaction can similarly lift degeneracy, induce a rugged landscape, and, thus, produce glassy dynamics.
\end{abstract}

DOI: 10.1103/PhysRevX.7.021030

\section{INTRODUCTION}

Geometrical frustration occurs widely in crystalline materials such as water ices, spin ices, magnets, and high-temperature superconductors [1]. Their lattice geometries induce conflicts between the interaction energies of neighboring atoms. Figure 1(a) illustrates the simplest geometrical frustration: three neighboring antiferromagnetic Ising spins on a triangular lattice (AFIT) cannot be simultaneously antiparallel to minimize their interaction energy [2]. Such geometrical frustration leads to many exotic behaviors including highly degenerate ground states, strong fluctuations, spin liquids, and various artificial particles, such as magnetic monopoles in spin ices [3,4]. Geometrical frustration has traditionally been studied using ensemble averaging techniques such as neutron and $\mathrm{x}$-ray scattering, muon spin rotation, nuclear magnetic resonance, and calorimetry [5]. To directly observe individual spins, arrays of micrometer-sized magnets [6,7], superconducting rings [8], and colloidal particles [9-12] have been used to mimic frustrated spins. In this rapidly evolving field of artificial geometrically frustrated systems [7], colloids have an advantage in directly visualizing the thermal motions of

Published by the American Physical Society under the terms of the Creative Commons Attribution 3.0 License. Further distribution of this work must maintain attribution to the author(s) and the published article's title, journal citation, and DOI.
Subject Areas: Condensed Matter Physics, Magnetism, Soft Matter individual spins, but the related experiments are limited [10-12]. We have demonstrated that a buckled colloidal crystal can mimic AFIT with measurable single-spin dynamics [10]. In the present work, we reveal the glassy dynamics at low effective temperatures in this system. The buckled colloidal crystals provide a simple platform for exploring the nature of glass transition [13], which in turn casts new light on geometrically frustrated materials at low temperatures.

Low-temperature spin dynamics is a key challenge in geometrical frustrated materials [16]. Unlike frustrations in structural glasses, which lead to rugged energy landscapes and glassy dynamics, geometrical frustrations on lattices give rise to numerous degenerated ground states which are well connected without high-energy barriers [5,17]. Therefore, spins should not have glassy dynamics. In real materials, however, inevitable subtle effects such as anisotropic interactions, long-range interactions, and lattice deformation can lift the degeneracy [1] and induce energy barriers, and, thus, could induce glassy dynamics at low temperatures. Glassy behaviors have been suggested by theoretic models $[18,19]$ and indirect experimental evidences in geometrically frustrated organic conductors [20] and magnetic compounds [21,22], which are considered unusual since glassy dynamics is generally associated with disordered systems rather than clean (i.e., defect-free and dopant-free) crystals or spin ices. Their microscopic mechanisms are poorly understood [20] because of a lack of 
(a)

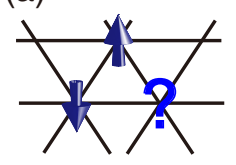

(b)

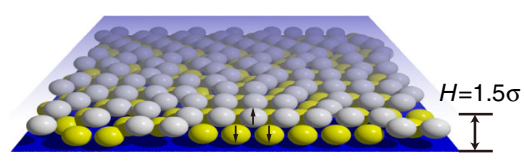

(c)

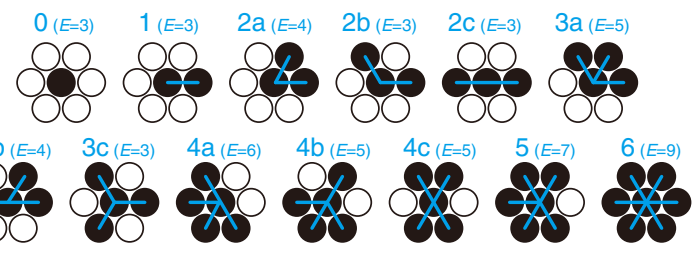

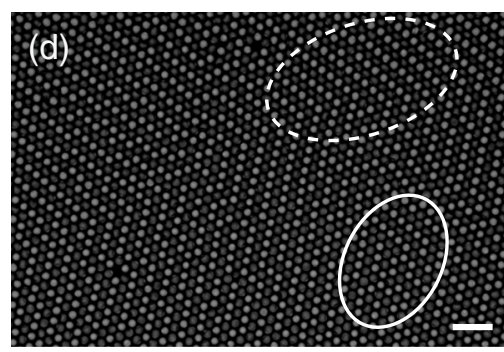
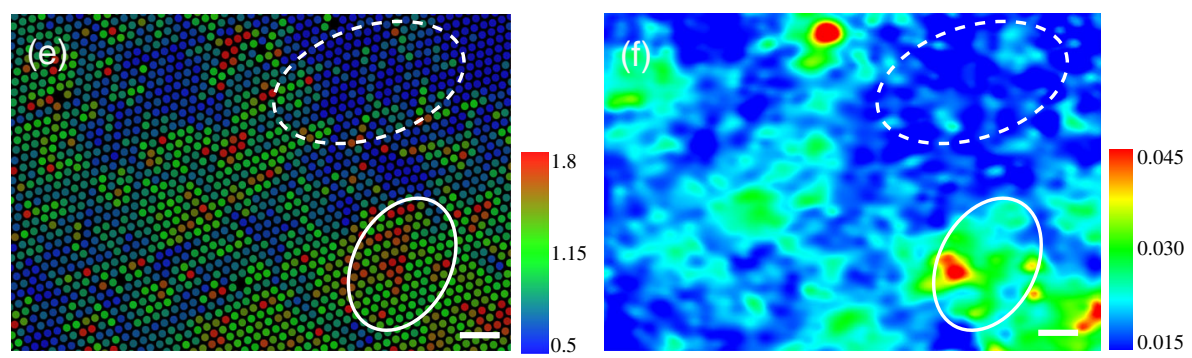

FIG. 1. Correlations among structures, soft vibrational modes, and dynamics in the buckled attractive colloidal crystal. The simulation counterpart is Fig. S4 of SM [14]. (a) Three spins on a triangular plaquette cannot simultaneously satisfy all antiferromagnetic interactions. (b) Schematic of the 1.5-layer colloid confined between two walls with spheres buckled up (white) and down (yellow). (c) All 13 possible local spin configurations, i.e., motifs, labeled with the number of frustrated bonds (blue lines) attached to the central spin. The motif's energy $E$ is the number of frustrated bonds. The weight of a bond at the edge is $1 / 2$ because it is shared between two motifs; hence, $E=6 / 2$ for motif $0, E=1+4 / 2$ for motif 1 , and so on. Panels (d)-(f) are the same sample region. Scale bar: $10 \mu$ m. (d) The raw image with bright spheres buckled up and dark spheres buckled down. (e) The color-coded mean-square displacement (MSD) of each particle normalized by the ensemble-averaged value. (f) The color-coded total magnitude of soft low-frequency modes whose participation ratio $p<0.2$ [15]. The dashed ellipses mark an ordered region in (d) which has weak motions in (e) and fewer soft modes in (f); the solid-line ellipses mark a disordered region in (d) which has stronger motions in (e) and more soft modes in (f).

microscopic measurements. Although artificial spin ices [7] provide a single-spin resolution for structures, the glassy dynamics has rarely been explored.

Here, we directly measure the glassy dynamics at the single-spin level using colloidal spheres. We pack monodispersed spheres in a 1.5-layer crystalline structure in the $x y$ plane and frustration-induced disorder in the $z$ direction, which can mimic Ising spins [Fig. 1(b)]. Such a partially ordered colloidal crystal avoids complex local configurations in structural glasses and the motions of all particles can be easily tracked. Thus, the glassy behaviors at the microscopic scale can be better elucidated. Based on the two novel features of the system for the glass studies, namely, the simple local structures and spinlike particles, we focus on two aspects. The first is the structural feature of dynamical heterogeneity (DH), i.e., the structure-dynamics relation in glassy frustrated spins. When a liquid is supercooled to a glass, its static structure does not change much, but the dynamics slows down drastically. To understand this puzzle, great efforts have been made to search for the structural origin of dynamic slowing-down in amorphous materials [23]. We aim to reveal such origin in the frustrated spin system. The second is the role of dynamic facilitation (DF) in spin relaxation. Although DF is purely about kinetics, it does not rule out a role for structure [23]. In fact, it is highly possible that certain regions are predisposed to relax more actively and facilitate relaxations further with certain structural features [23]. We confirm the dynamic facilitation in this frustrated spin system and clearly resolve the spacetime structures of spin excitations.
More importantly, we find the connection between local spin order and facilitation, which is beyond the description of DF.

The content of the paper is as follows. We first introduce the experiment and the AFIT model. Figure 1 depicts the system and shows the structure-dynamics correlations. Figures 3 and 4 characterize the glassy dynamics as the main contribution of this paper, i.e., the first microscopic observation of glassiness in geometrically frustrated lattices. Figures. 5-9 are about the role of facilitation on spin relaxation. This paper bridges two fields (geometrical frustration and glassy materials) and is related to Coulomb liquid in statistical physics and colloid in softmatter physics; we therefore provide more explanations in the Supplemental Material (SM) [14] for readers with different backgrounds.

\section{EXPERIMENT}

We use two types of aqueous colloidal suspensions. One is repulsive poly( $N$-isopropylacrylamide) (NIPA) microgel spheres with short-ranged repulsions [24] [Fig. S1(a) of SM [14] ]. Their diameter $\sigma$ decreases linearly with temperature from $0.75 \mu \mathrm{m}$ at $24.5^{\circ} \mathrm{C}$ to $0.67 \mu \mathrm{m}$ at $28.5^{\circ} \mathrm{C}$ [Fig. S1(b) of SM [14] ]; thus, the volume fraction $\phi$ is tunable. For colloidal hard spheres, $\phi$ plays a similar role to the inverse effective temperature $1 / T_{\text {eff }}$ of atomic systems. The second is attractive poly(methyl methacrylate) (PMMA) spheres with a hard core of $2.08 \mu \mathrm{m}$ in diameter. At high temperatures, the attraction is stronger [25] [see Fig. S2(b) of 
SM [14] ]; i.e., $T_{\text {eff }}$ is lower. When the NIPA or PMMA spheres are confined between two parallel walls $1.5 \sigma$ apart, they assemble into a buckled triangular lattice [Fig. 1(b)]. Half of the spheres in the focal plane are bright, and the other half, located near the bottom wall, are slightly out of focus and appeare dark [Fig. 1(d)]. The neighbors of a sphere tend to be in the opposite Ising state for more free-volume entropy, i.e., lower free energy. Therefore, neighboring spheres have an effective antiferromagnetic interaction and the buckled crystal is analogous to AFIT, which is a prototype model of geometrical frustration. Colloidal spins actively flip at high $T_{\text {eff }}$, (i.e., weak antiferromagnetic interaction or low $\phi$ ) (Movie 1 of SM [14]) and jam at low $T_{\text {eff }}$ (Movie 2 of SM [14]). We tune the temperature using an objective heater and record the Brownian motions of $\sim 2500$ NIPA or $\sim 3500$ PMMA spheres within larger crystal domains under equilibrium using a charge-coupled device camera. The positions of particles are tracked using standard image analysis [26]. Details of the experiments and the corresponding simulations are provided in the Supplemental Material [14].

\section{AFIT MODEL}

In the AFIT model, one spin has 13 possible nearestneighbor configurations (i.e., motifs), as shown in Fig. 1(c). Ground states allow only motifs $0,1,2 b, 2 c$, and $3 \mathrm{c}$ because each of their triangular plaquettes contains only one frustrated bond [Figs. 1(c) and 10(a)]. AFIT is an exact solvable model with degeneracy $\Omega=e^{0.323 N}$ and entropy $S=k_{B} \ln \Omega=0.323 k_{B} N$ for $N$ spins at ground states [27], where $k_{B}$ is the Boltzmann constant. For the colloidal system, a lower $T_{\text {eff }}$ induces stronger buckling and antiferromagnetic interactions, which causes the inplane triangular lattice to deform slightly. Therefore, the deformable AFIT model better describes the buckled crystal at high packing fraction. The satisfied bonds projected into the $x y$ plane are approximately $3 \%$ shorter on average than the frustrated bonds in both repulsive [10] and attractive buckled crystals. Hence, a ground-state plaquette featuring one frustrated bond and two satisfied bonds is an isosceles triangle, which can tile the 2D space with only motifs $2 \mathrm{~b}$ and $2 \mathrm{c}$ to form random zigzag stripes [Fig. 10(b)] [10]. All the $e^{0.323 N}$ ground states of AFIT are fully connected by the barrier-free flipping of motif $3 \mathrm{c}$ [17], whose flip maintains the same number of frustrated bonds without changing the energy. Hence, AFIT should not have glassy dynamics. By contrast, the slight lattice deformation relieves most ground states of AFIT; none of the ground states of deformable AFIT (i.e., zigzag stripes) contain motif $3 \mathrm{c}$ and their transformation requires the collective motion of $\sim \sqrt{N}$ particles [Fig. 10(c)], which corresponds to a high-energy barrier and, thus, leads to glassy dynamics. Details about AFIT and deformable AFIT structures are in Appendix C.

\section{STATIC STRUCTURES AND VIBRATIONAL MODES}

Structural glasses are disordered in spaces with a continuous degree of freedom; hence, they have complex local structures that are difficult to characterize and distinguish. For example, a particle in the 3D glass of monodispersed hard spheres has numerous coordination polyhedrons [28]. By contrast, the buckled crystal is mainly disordered in the $z$ direction with two possible Ising states for each sphere; hence, a sphere has only 13 motif structures, as shown in Fig. 1(c). Such simple local structures enable us to develop clear structure-dynamics correlations.

As the packing fraction increases, the colloidal crystal forms more ordered buckled stripes due to the spin-lattice coupling [10]. Such spin structure can be characterized by the spatial spin correlations $C_{r}$ shown in Fig. S3 of SM [14]. $C_{r}$ of perfect zigzag stripes (i.e., the ground states of deformable AFIT) have long-range correlations, in contrast to the quasi-long-range correlation for the ground state of AFIT. For the colloidal crystals, the spin correlation length increases at lower $T_{\text {eff }}$ up to several lattice constants (Fig. S3 of SM [14]), which corresponds to the typical size of domains containing parallel stripes [dashed ellipse in Fig. 1(d)]. This is similar to the length of short-range order (SRO) found in the metallic [28] and colloidal [29] glasses, but the SRO of the stripe domains in Fig. 1(e) can be easily visualized.

Besides the real-space structure, another static characterization is the vibrational modes. We extract the normal modes by diagonalizing the covariance matrix of the particles' in-plane displacements [30,31]. The participation ratio defined as $p(\omega)=\left(\sum_{i}\left|\vec{e}_{\omega, i}\right|^{2}\right)^{2} /\left(N \sum_{i}\left|\vec{e}_{\omega, i}\right|^{4}\right)$ is used to measure the degree of localization of each mode. $p(\omega)=1 / N$ when the vibrational mode is fully localized at one single particle; $p(\omega)=1$ corresponds to plane waves without localization. The contribution from quasilocalized soft modes is measured by the participation fraction $p_{i(<0.2)}=\frac{1}{N_{0.2}} \sum_{p(\omega)<0.2}\left(e_{\omega, i}\right)^{2}$, where $N_{0.2}$ is the number of modes whose $p(\omega)<0.2$ [15]. Examples of the normal modes with different degrees of localizations are shown in Fig. 2. Low-frequency modes with relatively low $p$ exhibit vortices featuring larger polarization vectors [Fig. 2(b)] and tend to localize in disordered regions [Fig. 1(d)]. By contrast, high-frequency modes are localized and composed of random vectors [Fig. 2(c)]. The strength of quasilocalized soft modes is shown in Fig. 1(f). Red regions in Fig. 1(f) are similar to soft spots in structural glasses, which can be regarded as structural defects that determine particle rearrangements in glasses [32]. Soft regions featuring quasilocalized modes are highly correlated with zones where particles are irreversibly rearranged in colloidal [31] and metallic [33] glasses. But structural features of soft spots have not been observed experimentally before. Here, we find that the quasilocalized soft modes in Fig. 1(f) are 

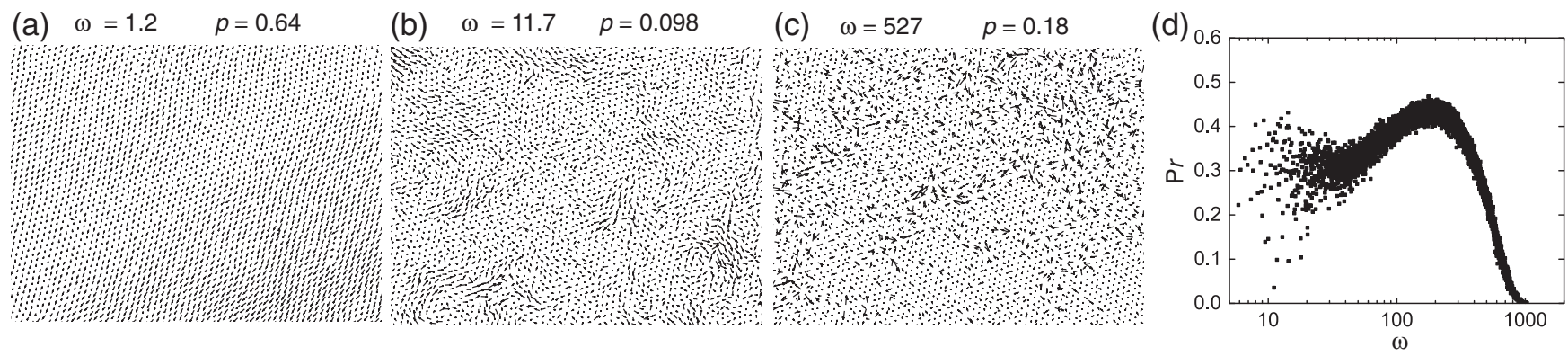

FIG. 2. Typical normal modes. (a) The nonlocalized plane wave with the lowest frequency $\omega$. (b) A quasilocalized mode with a low $\omega$ and a low participation ratio $p$. (c) A mode with a high $\omega$ and low $p$. The polarization vectors in (a)-(c) show the vibration amplitude and direction of individual particles. (d) Participation ratios of all modes.

highly correlated with the disordered regions with highenergy motifs in Fig. 1(d), which is in accordance with simulation results showing that the motifs in soft regions are not geometrically favored in metallic glasses [33].

In glass-forming liquids composed of polydispersed spheres, patches with a higher crystalline order, often interpreted as medium-range crystalline order $[29,34]$ or SRO [28], contribute more to slow dynamics. Here, we find a similar result that the slow dynamics characterized by a smaller mean-square displacement (MSD) in Fig. 1(e) are strongly correlated with the ordered stripes in Fig. 1(d). Moreover, they are anticorrelated with the soft regions in Fig. 1(f). The 3D normal modes and MSD along the $z$ direction also exhibit the same correlations (Fig. S4 of SM [14]). These obvious correlations among structures, soft modes, and dynamics in Figs. 1(d) and 1(f) and Fig. S5 of SM [14] demonstrate that motifs $2 b$ and $2 c$ contribute significantly to slow dynamics and nonsoft modes.

The ground states of deformed AFIT are random zigzag stripes corresponding to the complete tiling of the 2D space by isosceles triangles or equivalently the random stacking of one-dimensional ordered spins as shown in Fig. 10(b) $[10,35]$. In experiments, however, we observe more ordered zigzag stripes having a persistence length of approximately $5 \sigma$ at low $T_{\text {eff }}$ [Fig. 1(d) herein and Fig. S4 of the SM [14] ], rather than the mean persistence length of $3 \sigma$ of random zigzag stripes (see Supplemental Material [14]). Such order induced by thermal fluctuations is a manifestation of the order-by-disorder effect in geometrical frustration [Fig. 10(d)] [35]. Softer fluctuations around a particular ground state can access a larger region of phase space. Consequently, the system spends more time around this state. Meanwhile, soft fluctuations tend to bring some degrees of long-range order into the frustrated system, i.e., order by thermal or quantum fluctuations [5]. Note that very long parallel stripes should not form since they will induce anisotropic shape deformation associated with large strain energy. Therefore, medium-sized stripe domains with alternative orientations are most energy favorable because strains are canceled out, just like martensitic structures in polycrystals [Fig. 10(e)].

\section{GLASSY DYNAMICS IN BUCKLED CRYSTALS AND COULOMB LIQUIDS}

In geometrical frustration, both spin-lattice coupling and long-range interaction [18] can relieve degeneracy; hence, they may similarly induce rugged energy landscape and glassy dynamics. In this section, we compare the glassy dynamics of the buckled colloidal spheres with short-range interactions on a deformable triangular lattice and Coulomb charges with long-range $1 / r$ potentials on a nondeformable triangular lattice.

A basic characterization of spin dynamics is the autocorrelation $C(t)=\left\langle s_{i}(0) s_{i}(t)\right\rangle$, where $s_{i}(t)= \pm 1$ refers to the Ising state of spin $i$ at time $t$ and the angle brackets are the ensemble average over all spins. The stretched exponential function $C(t)$ [Fig. 3(b)] and the sharply increasing relaxation time $\tau$ at lower temperatures [Fig. 3(b), inset] reflect the slowing down of dynamics. The change of superArrhenius to Arrhenius behavior in the inset of Fig. S5(b) indicates a fragile to strong crossover. The fragile-to-strong crossover has also been found in the simulation of hard disks confined in quasi-one-dimension [36]. The stretched exponential decay is often interpreted as a superposition of many simple relaxations with different exponential decays [37]. Consequently, we further measure the persistence functions $P(t)$ of different motifs, which indeed vary dramatically due to their different energies [Fig. 3(c)]. However, $P(t)$ are still stretched exponential rather than exponential, indicating that the same type of motifs have different stabilities in different local environments, in accordance with the collective nature of the free volume in sphere packing.

A recent simulation discovered that the Coulomb liquid can form glassy stripes at low temperatures [38]. Here, we further measure $C(t)$ and $P(t)$ of each type of charge motifs in Figs. 3(e) and 3(f), which exhibit similar stretched exponential decays to those of the buckled colloidal crystal. But the Coulomb liquid exhibits Arrhenius behavior without a crossover to super-Arrhenius behavior [Fig. 3(e), inset] since the glassy dynamics is dominated by local charge hopping rather than cooperative motions [39]. 

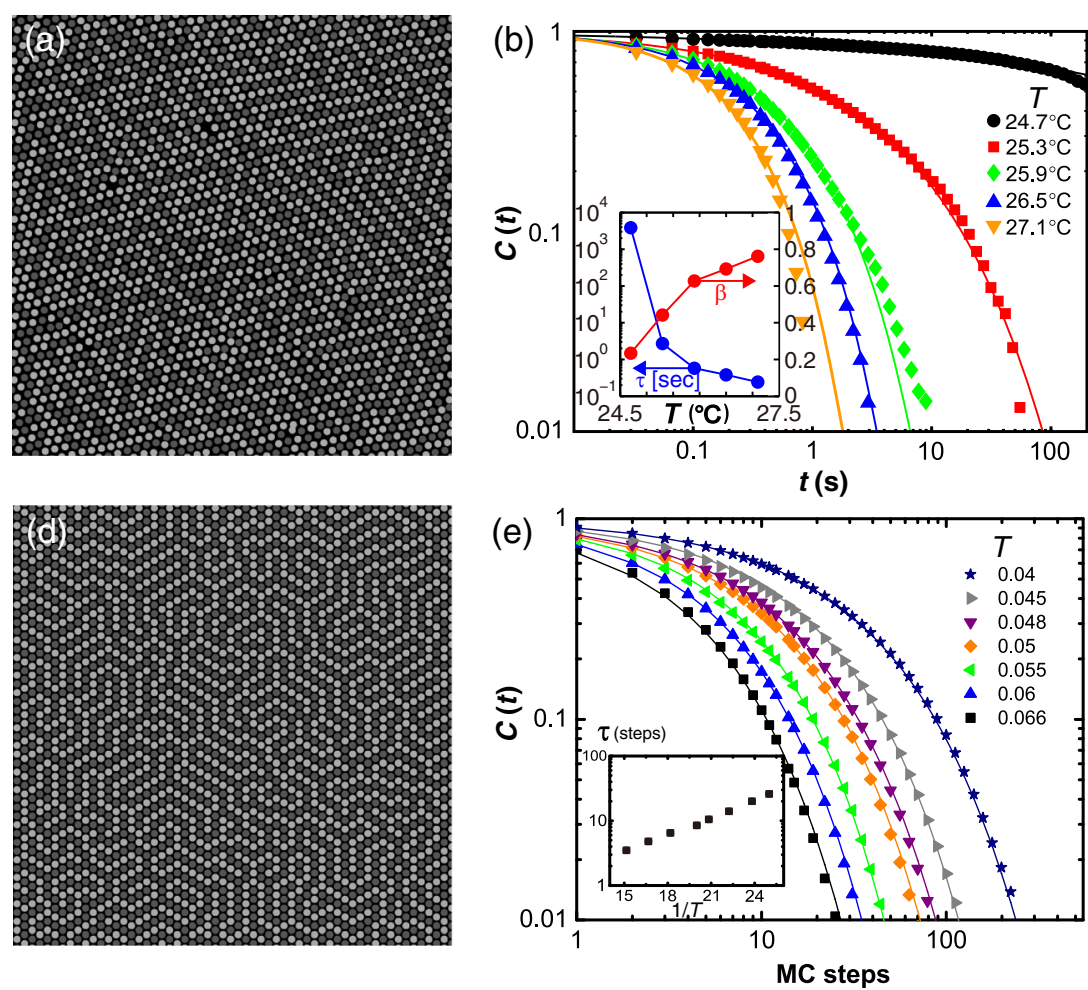
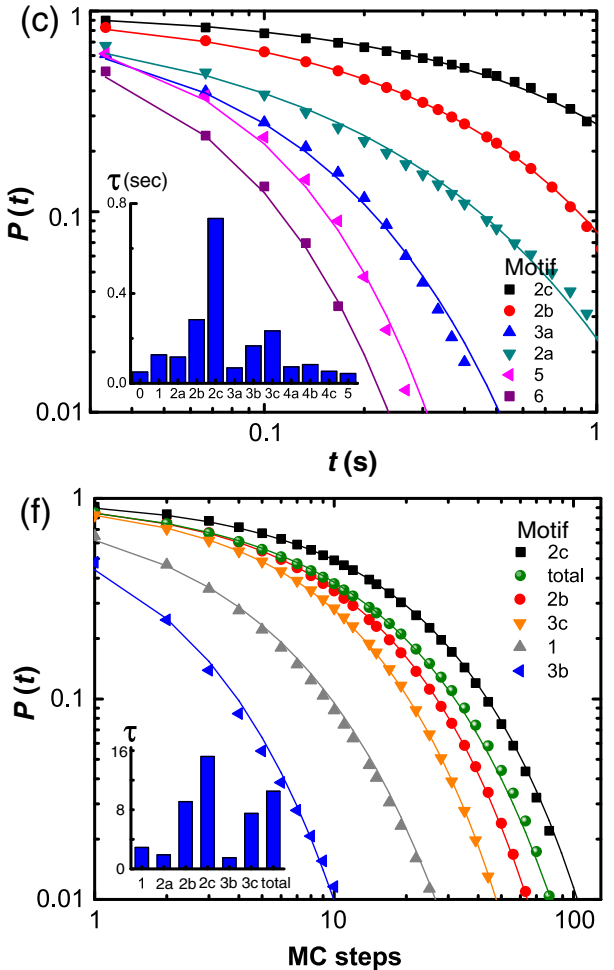

FIG. 3. The dynamics of the repulsive buckled crystal in (a)-(c) and the Coulomb liquid in (d)-(f). The simulation counterpart of (a)-(c) is Fig. S5 of SM [14]. (a),(d) Typical real-space structures. Coulomb charges (white spots) fill half of the triangular lattice. (b),(e) Spin or charge autocorrelation function $C(t)$ ensemble averaged over all motifs. Panel (b) is reproduced from Ref. [10] with permission, copyright 2008, Nature Publishing Group. (c),(f) Persistence function of each type of motif. This function describes the fraction of motifs that have not changed state during time $t$. Solid curves in (b),(c),(e),(f) are stretched exponential fits, exp $\left[-(t / \tau)^{\beta}\right]$, with the relaxation time $\tau$ shown in the insets. Temperature $T=25.3{ }^{\circ} \mathrm{C}$ in (a),(c) and $T=0.04$ in (d),(f).

Similar Arrhenius behavior was experimentally observed in the charge-cluster glass [39], which can be modeled as lattice Coulomb liquid [38].

In Sec. III, we attribute the glassiness as a result of spinlattice coupling which requires a collective flip of $\sqrt{N}$ spins to change from one ground state to another ground state. For low-temperature nonground states, the relaxation should require the collective flips of many spins less than $\sqrt{N}$ [see, e.g., Figs. 10(b) and 10(c)]. By contrast, the Coulomb liquid with no spin-lattice coupling but longrange interactions at low temperatures contains not only motifs $2 \mathrm{~b}$ and $2 \mathrm{c}$ but also motifs 1 and $3 \mathrm{c}$. They all belong to the ground state of AFIT, and nearest-neighbor interactions are the same. However, different configurations of farther neighbors induce slightly different energies due to the longer-ranged interactions and produce a rugged energy landscape with many basins slightly higher than the true ground states (i.e., perfect parallel stripes). Therefore, spinlattice coupling and long-range interaction have similar effects on the glassy behaviors.

Approaching the glass transition, the dynamics of the liquids becomes not only progressively slower but also more spatially heterogeneous. Such dynamical heterogeneity plays a central role in the description of glass-forming liquids and has been intensively studied in metallic, molecular, and colloidal systems [13], but not yet in geometrically frustrated lattices. DH in spacetime is usually characterized by the four-point correlation function of mobility for structural glasses. Here, we measure the analogous four-point correlation function of spin flips [40]:

$$
C_{4}(r, t)=\frac{\left\langle a_{i}\left(t, t_{0}\right) a_{i+r}\left(t, t_{0}\right)\right\rangle-\left\langle a_{i}\left(t, t_{0}\right)\right\rangle\left\langle a_{i+r}\left(t, t_{0}\right)\right\rangle}{1-\left\langle a_{i}\left(t, t_{0}\right)\right\rangle^{2}},
$$

where $a_{i}\left(t, t_{0}\right)=s_{i}\left(t_{0}\right) s_{i}\left(t+t_{0}\right)$. The second term and the denominator scale $C_{4}$ in $[0,1]$. The dynamic correlation $C_{4}(r, t=\tau)$ of spin flips in Fig. 4(a) and the static correlation of spins in Fig. S3 of the SM [14] have similar exponential decays and correlation lengths, indicating a correlation between dynamics and structure. The four-point susceptibility, $\chi_{4}(t)=\sum_{r} C_{4}(r, t)$, probes the correlation strength and the degree of $\mathrm{DH}[13] \cdot \chi_{4}(t)$ in Fig. 4(b) peaks higher and shifts to a longer time at low temperatures, reflecting the growing cluster size of correlated spin flips and progressively slower relaxations.

The single-spin resolution enables the direct visualization of $\mathrm{DH}$, which is beyond what $C_{4}$ and $\chi_{4}$ can describe. 

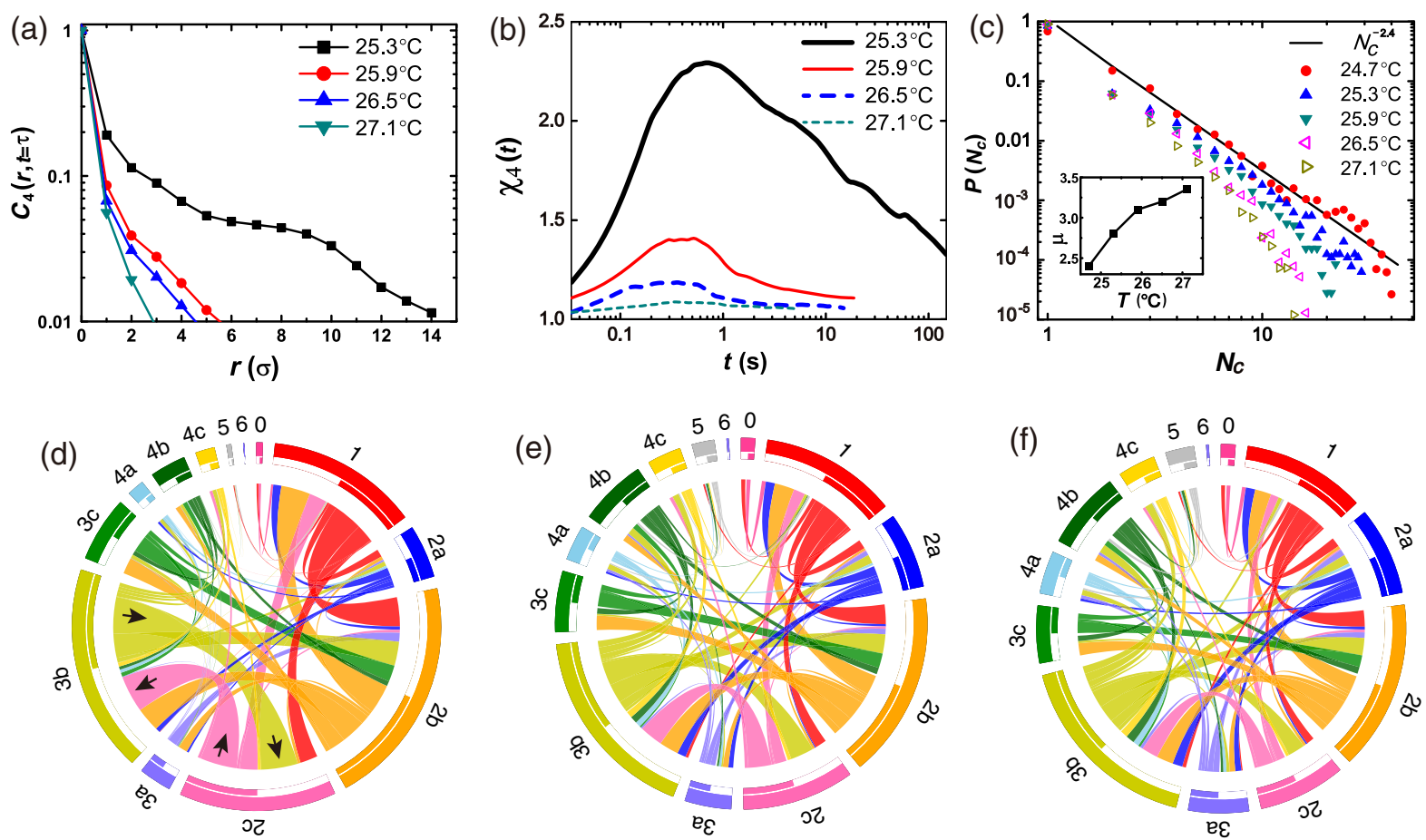

FIG. 4. Glassy dynamics in the repulsive buckled crystal. The simulation counterpart is Fig. S6 of SM [14]. (a) Four-point spin correlation function $C_{4}(r, t=\tau)$. (b) Dynamical four-point susceptibility $\chi_{4}(t)$. (c) The probability distributions of the cluster size $N_{c}$ of the $10 \%$ most flipped spins at different temperatures. The fitting line $P\left(N_{c}\right) \propto N_{c}^{-\mu}$, where $\mu$ is shown in the inset. (d)-(f) Statistics of the transition events among 13 motifs shown by circular flow plots at (d) $24.7^{\circ} \mathrm{C}$, (e) $25.3^{\circ} \mathrm{C}$, and (f) $27.1^{\circ} \mathrm{C}$. The length of the outer thick arc is proportional to the total number of spin flips from or towards the labeled motif number. The flips from the motif are labeled by the color of the motif arc. Some inflows and outflows between motifs $2 \mathrm{c}$ and $3 \mathrm{~b}$ are labeled with arrows as examples. The inner thin arc length is proportional to the number of flips from the motif, and is half the length of its corresponding outer thick arc. Hence, the incoming and outgoing flows are equal, as required by the detailed balance under equilibrium.

Figure S7 of SM [14] shows that the most frequently flipped $10 \%$ of spins during the relaxation time $\tau$ are dispersed at high $T$ but form compact clusters at low $T$, reflecting an increasing degree of DH. The cluster size of the most-flipped spins follows a power-law distribution $P\left(N_{c}\right)=N_{c}^{-\mu}$, where $\mu$ increases with $T$ [Fig. 4(c)] [41]. The power-law distributions of fast-moving particles with a similar $\mu$ have been observed in supercooled liquids composed of Lennard-Jones particles [42], colloidal spheres [41], and ellipsoids [43]. Compared with these structural supercooled liquids with complex local structures, the spin system with only 13 motifs enables us to resolve the detailed dynamics among motifs.

The transitions between the 13 motifs are exhaustively shown by the circular flow plots in Figs. 4(d)-4(f). Note that Figs. 4(d)-4(f) show only the statistics of flipping events. At low $T\left(24.7^{\circ} \mathrm{C}\right)$, there are only $0.7 \%$ of the unstable motifs, i.e., motifs $4 \mathrm{a}, 4 \mathrm{~b}, 4 \mathrm{c}, 5$, and 6 , but they contribute to approximately $8 \%$ of the flipping events [Fig. 4(d)]. Figures 4(d)-4(f) show that transformation mainly occurs between motifs with low free-energy barriers. For example, motif 1 often transforms to motif $2 b$ or $2 \mathrm{c}$, but rarely to motif $3 \mathrm{~b}$. Instead, motif 1 transforms to motif $2 b$ or $2 c$ first before further transforming to motif $3 b$, especially at low $T_{\text {eff }}$. This hierarchical dynamics is reminiscent of the microscopic dynamics observed in structural glassy systems: dynamics at large length scales is a combination of those at small length scales because of the growing activation free-energy barriers $[44,45]$. By contrast, the discrete degrees of freedom in the AFIT model allows constructing its state space as a network whose state connections can directly reflect the hierarchical dynamics [17]. The circular plots in Figs. 4(d)-4(f) reveal connections between $\mathrm{DH}$ and the motif structures, but cannot show how spin flips are correlated in spacetime, which is the key topic in DF and is discussed in the next section.

\section{FACILITATED DYNAMICS}

We further study the role of facilitation in buckled colloidal crystals. Kinetically constrained models emphasize a purely kinetic picture of glass transition in which spins flip according to local kinetic constraints. Because of these constraints, dynamic excitations can be triggered only by recent excitations nearby, a phenomenon known as dynamic facilitation [46]. The facilitated dynamics and the scarcity of excitations lead to hierarchical and cooperative relaxation. Recently, spin-based DF has been generalized to 
structural glasses [44] and examined in colloidal glasses [47-49]. Here, we confirm the facilitation in spin systems and its importance for spin relaxation in spacetime.

Figure 5 shows that the flipped spins disperse in space at high $T$ and coalesce into clusters (i.e., DH) at low $T$. Compared with the first $10 \%$ of particles that ever flipped in Fig. 5, later excitations (e.g., green particles belonging to the top $30 \%$ but not the top 10\%) tend to occur adjacent to earlier ones. This illustrates that further spin flips are facilitated by recently flipped ones.

A comprehensive description of DH requires information about the geometry of dynamics in spacetime, which is beyond the characterizations in Figs. 4 and 5. The geometry of dynamics in spacetime is profoundly shaped by facilitation, which is the idea behind DF. Since most excitations are triggered by previous ones, mobile regions form a connected structure as a bundle of excitation lines in spacetime which coalesce, branch, and percolate [46]. The excitation lines are embedded in the immobile regions called spacetime bubbles [45]. In our system, an excitation is a spin flip. We color the excitations in blue over a coarsegraining time for better visualization in Fig. 6. Different choices of coarse-graining time yield similar results (Fig. S8 of SM [14]). Excitations are randomly dispersed in spacetime at high $T$ [Fig. 6(a)], indicating a nonglassy liquid without facilitation and DH (Movie 3 of SM [14]). At low $T$, by contrast, excitations coalesce into sparse strings directed in time and form bundles [Fig. 6(b)], because longlived excitations facilitate the formation of nearby excitation lines (Movie 4 of SM [14]). The density of excitations increases with temperature as expected [Fig. 6(c)]. The stronger DF at lower temperatures naturally explains the enhanced DH. Moreover, the density of unstable motifs similarly increases with temperature [Fig. 6(c)], indicating a correlation between the static structures and the dynamic excitations. Indeed, the dynamic excitations mainly occur at the high-energy motifs, i.e., the nonstriped regions marked by the solid-line ellipses in Fig. 5. This is beyond the pure dynamical theory of DF, but makes intuitive sense and is consistent with the structure-dynamics correlations shown in Fig. 1.

In DF, local relaxations occur on two fundamental time scales: the exchange time $\tau_{x}$ between two consecutive flips of a spin and the persistence time $\tau_{p}$ for a spin to flip for the first time from a random starting time [45]. $\tau_{x}$ and $\tau_{p}$ are similar with the same distribution in normal liquids, but are distributed differently in glassy liquids [45]. The decoupling of $\tau_{x}$ and $\tau_{p}$ explains the DH and the breakdown of the Stokes-Einstein relation $D_{s} \propto \eta^{-1}$, where $D$ is the self-diffusion constant proportional to $1 / \tau_{x}$, and $\eta$ is the
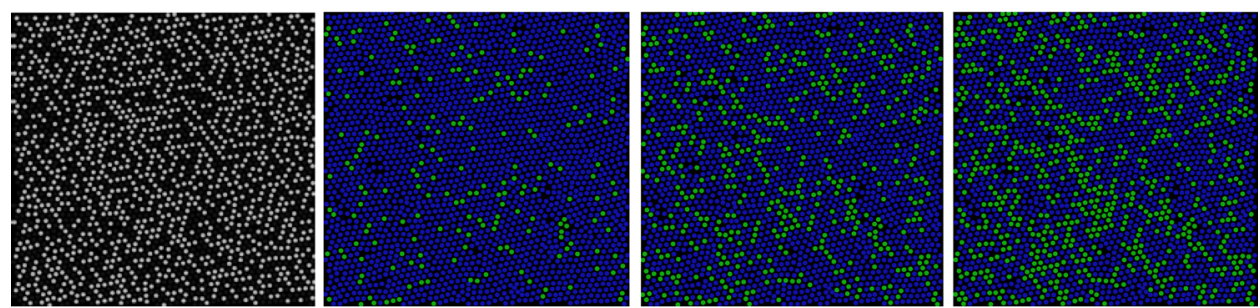

$27.1^{\circ} \mathrm{C}$
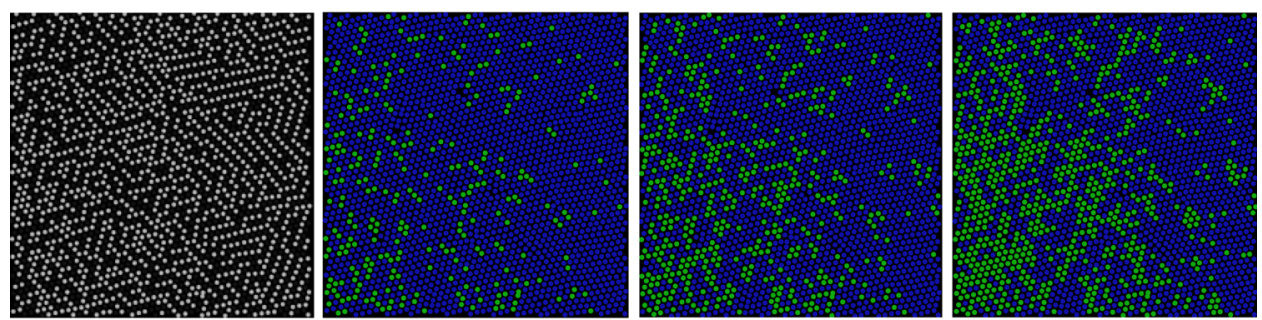

$25.3^{\circ} \mathrm{C}$
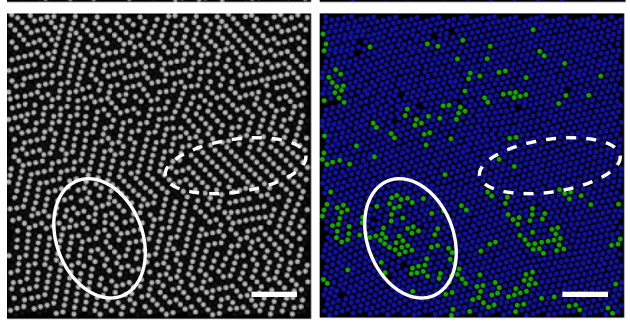

$10 \%$

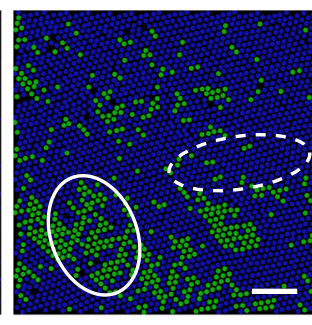

$20 \%$

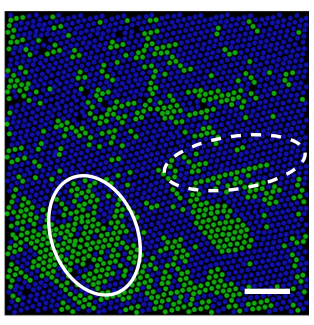

$30 \%$

FIG. 5. Repulsive NIPA colloidal crystals at the three temperatures indicate stronger facilitated dynamics at lower $T$, i.e., higher $\phi$. The first column shows the buckled crystals at different temperatures. The first 10\%, 20\%, and 30\% of flipped spins are colored in green in the last three columns, respectively. They show that the newly flipped spins are mainly around the previously flipped spins, i.e., facilitation. Dashed-line and solid-line ellipses at $24.7^{\circ} \mathrm{C}$ mark an ordered and a disordered region, which correspond to nonexcited and excited regions, respectively. 
(a)
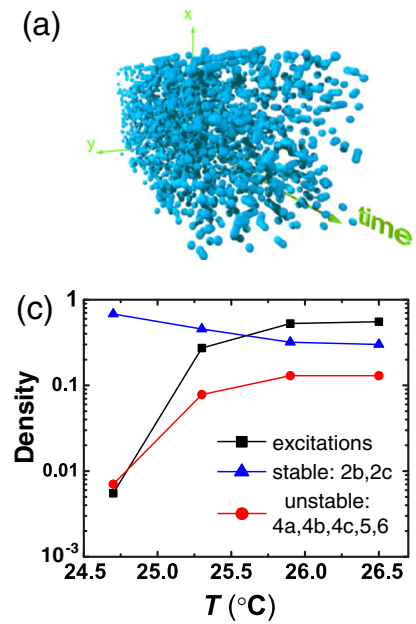

(b)
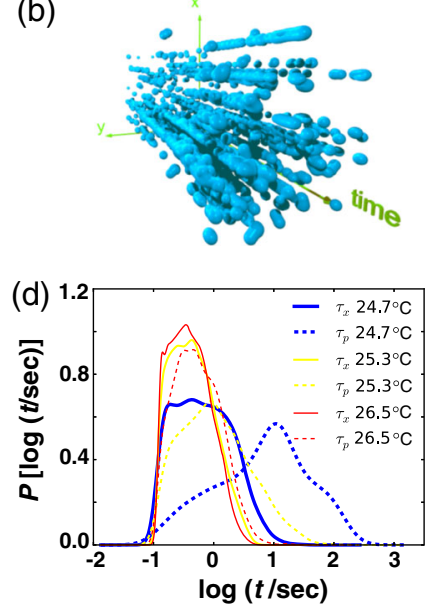

FIG. 6. Dynamic facilitation in the repulsive buckled crystal. (a),(b) Actively flipping regions labeled in blue in the $(32 \mu \mathrm{m})^{2} \times$ $4 \mathrm{~s}$ spacetime at $27.1^{\circ} \mathrm{C}$ and $(32 \mu \mathrm{m})^{2} \times 200 \mathrm{~s}$ spacetime at $24.7^{\circ} \mathrm{C}$, respectively. The excitation coarse-graining time is $0.067 \mathrm{~s}$ in (a) and $0.33 \mathrm{~s}$ in (b). Different coarse-graining times yield similar spacetime structures, but shorter times make the structure too crowded to be clearly visualized. Excitations dispersed in spacetime at high $T$ in (a) and coalesced into clusters at low $T$ in (b). (c) Densities of the excitations, unstable, and stable motifs. (d) Probability distributions of $\tau_{x}$ and $\tau_{p}$. $\left\langle\tau_{x}\right\rangle<\left\langle\tau_{p}\right\rangle$ below $26.5^{\circ} \mathrm{C}$, which signals the onset of $\mathrm{DH}$.

viscosity proportional to $\tau_{p}$ [50]. Despite their importance, $\tau_{x}, \tau_{p}$, and their distributions have rarely been measured in real glassy materials because of a lack of measurable single-spin dynamics. Although DF has been generalized to structural glasses, the excitation lines, $\tau_{x}, \tau_{p}$, and their distributions are difficult to measure because a spin represents a mesoscopic region [46], which takes a very long time to relax. By contrast, a single particle in a buckled crystal represents a spin such that these quantities can be directly measured, as shown in Figs. 6(b) and 6(d).

A more quantitative measurement of facilitation is the mobility transfer function $F(\Delta t)$ [48,51,52], which measures the excessive probability that a neighbor of a mobile particle is facilitated compared with an immobile particle, i.e., the degree of facilitation. $F(\Delta t)=$ $\left(\int_{0}^{r_{c}} P_{F}(r) d r\right) /\left(\int_{0}^{r_{c}} P_{F}^{*}(r) d r\right)$ [51], where $r_{c}$ is first minimum of the radial distribution function. For two consecutive time intervals $\Delta t_{12}$ and $\Delta t_{23}, P_{F}(r)$ is the probability of finding the smallest distance $r$ between a newly mobile spin in $\Delta t_{23}$ and an existing mobile spin in $\Delta t_{12}$, while $P_{F}^{*}(r)$ is the probability of finding $r$ between a newly mobile spin in $\Delta t_{23}$ and a randomly chosen immobile spin in $\Delta t_{12}$. Here, mobile spins are defined as the $10 \%$ most mobile ones during $\Delta t$. Figure 7(a) shows that the difference between $P_{F}(r)$ and $P_{F}^{*}(r)$ is greater near the relaxation time [51]. Consequently, $F(\Delta t)$ in Fig. 7(b) is higher around the relaxation time, suggesting that mobile spins over $\tau$ have stronger correlations. In addition, $F(\Delta t) \sim 1$ at high $T$
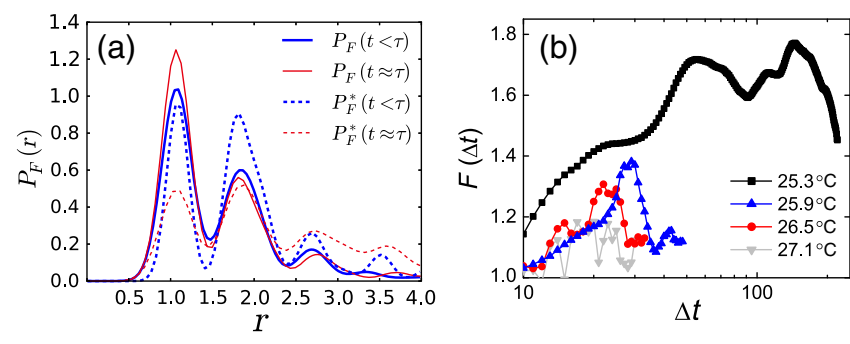

FIG. 7. Mobility propagation through facilitation. (a) The probability distributions $P_{F}(r)$ and $P_{F}^{*}(r) . P_{F}(r)$ and $P_{F}^{*}(r)$ for two time scales $(\Delta t<\tau$ and $\Delta t \approx \tau)$ are shown for comparison. (b) Mobility transfer functions at different temperatures.

(i.e., low $\phi$ ) in Fig. 7(b), indicating the absence of facilitation. The large $F(\Delta t)$ at low $T$ reflects a significant role of DF.

The dynamics of a spin depends not only on its neighbors in the closest layer, but also the ones farther away. Hence, finding an accurate structure-dynamics connection requires many parameters $X_{i}$ for describing numerous types of structures of multiple layers of neighbors. The individual spin dynamics, e.g., $Y=1$ for mobile spin, $Y=-1$ for immobile spin, can be measured in the highdimensional parameter space $\left\{X_{i}\right\}$. The machine-learning algorithm, support vector machine (SVM), provides an efficient way to quantify the effect of $X_{i}$ 's on $Y$ in this highdimensional space, e.g., finding a hyperplane which can best separate $Y=1$ and -1 points. This approach has recently been used to find the relation between local structures and relaxation in a structural glass [53]. Inspired by this work, here we explore the connection between spin relaxation and the local structure of four layers of neighbors using a training set containing 1000 particles. The functions, or features, of the structure are the average energies of its zeroth, first, second, third, and fourth layers of neighbors, i.e., $X_{1}, \ldots, X_{5}=E_{0}, \ldots, E_{4}$. They can be obtained from the motif energies described in Fig. 1(c). We label a mobile spin during $\Delta t_{12}$ with $Y=1$ if it makes one of its first-layer immobile neighbors become mobile during $\Delta t_{23}$. Otherwise, it is labeled with $Y=-1$. We employ the linear kernel of SVM [54] to construct the hyperplane that best separates the $Y=1$ and -1 points in the five-dimensional parameter space. The distance of each point to the hyperplane is defined as the softness $S$ (Ref. [53]), which reflects the propensity for relaxation. The distribution of $S$ for mobile spins in Fig. 8(a) shows that softer spins have a higher propensity for facilitation. In Fig. 8(a), the distribution of $S$ peaks at negative values for the ground-state motifs $2 b$ and $2 c$ and at large positive values for high-energy motifs. By contrast, the distributions of $S$ for different types of motifs are similar at high $T$, where DF vanishes [Fig. 8(b)], demonstrating that the local structure decouples from the dynamics and the softness fails to reflect the propensity for facilitation. In short, 


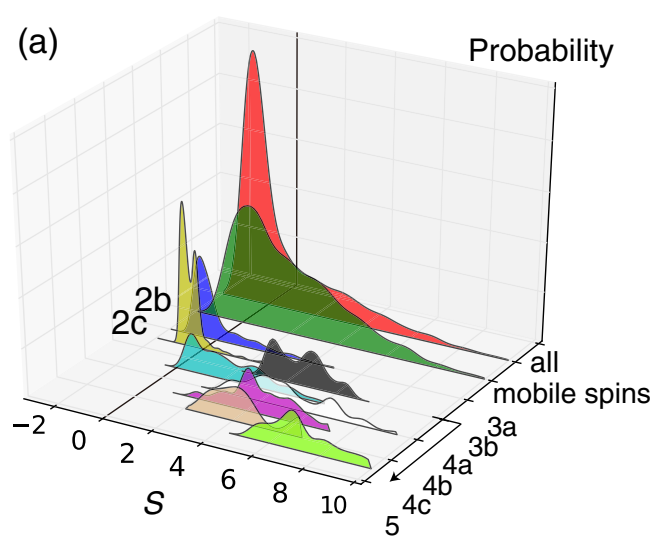

(b)

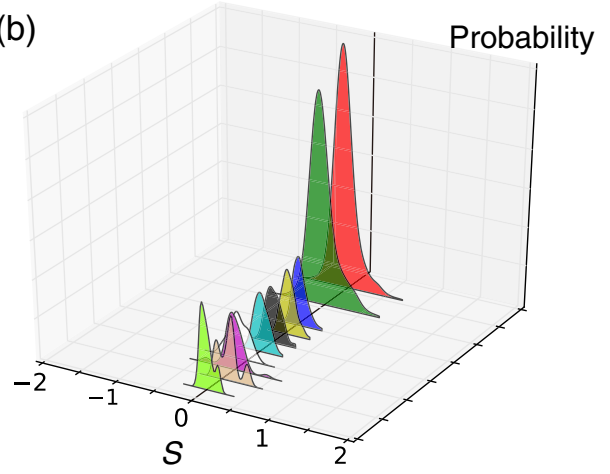

FIG. 8. Softness distributions. The distributions of $S$ of all spins, mobile spins with facilitation, and local motifs at (a) $25.3^{\circ} \mathrm{C}$ and (b) $27.1{ }^{\circ} \mathrm{C}$. The curves of local motifs are rescaled by $25 \%$ for clarity.

softness can accurately predict local structures with facilitation in the glassy regime.

Next, we apply the principal component analysis (PCA) to find five principal components $\mathrm{PC}_{1}, \ldots, \mathrm{PC}_{5}$ [55]. As an unsupervised approach, PCA analyzes a set of features $\left(E_{0}, \ldots, E_{4}\right)$ without their categories $(Y)$ and allows identifying correlations among principal components. The first and second largest principal components form a 2D subspace that separates spins in groups with higher energy and facilitation (motifs 4 and 5) from nonmobile ones (motifs $2 \mathrm{~b}$ and 2c). The PCA results in Fig. 9 without using categorical information are consistent with those in Fig. 8 using SVM, which supports our conclusion about the structural origin of DF. In addition, PCA measures the contribution of different neighboring layers to the first and second principal components, as shown in Fig. 9(c). As expected, the first layer of neighbors make the largest contribution, but other layers also contribute between $5.06 \%$ and $22.6 \%$.

\section{DISCUSSION}

This soft-matter system provides novel connections among geometrical frustration, glassy dynamics, and Coulomb liquid. Here, we show that spin-lattice coupling
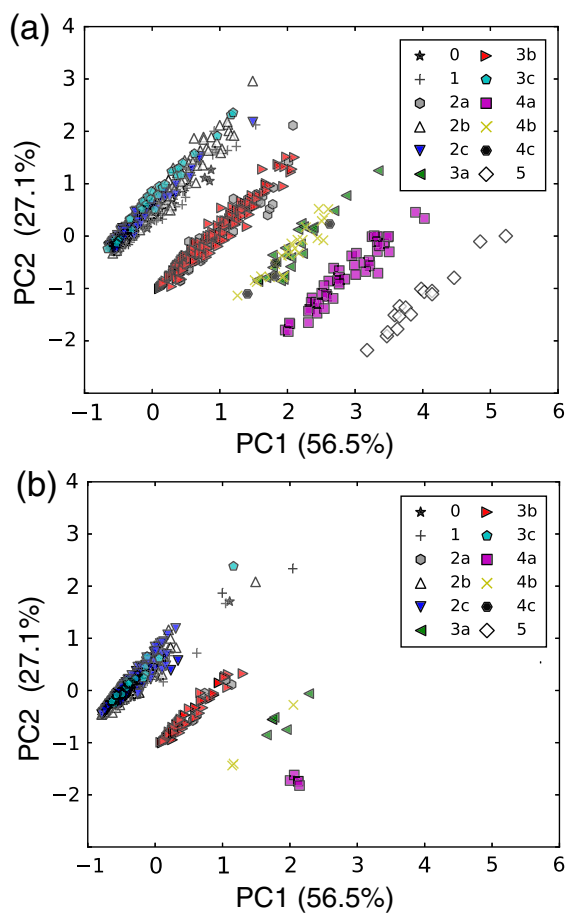

(c)

\begin{tabular}{|c|c|c|}
\hline & PC1 $(\%)$ & PC2 $(\%)$ \\
\hline$E_{0}$ & 54.87 & 34.06 \\
\hline$E_{1}$ & 22.64 & 1.23 \\
\hline$E_{2}$ & 9.79 & 31.3 \\
\hline$E_{3}$ & 7.64 & 23.93 \\
\hline$E_{4}$ & 5.06 & 9.48 \\
\hline
\end{tabular}

FIG. 9. Principal component biplots. The first two principal components for (a) mobile and (b) nonmobile spins at $25.3^{\circ} \mathrm{C}$. The percentages of explained variations for the first two principal components are $56.5 \%$ and $27.1 \%$, respectively. They are higher than the mean value of $20 \%$, reflecting a greater contribution to the spin dynamics. Symbols represent different motifs. The cluster distribution results from the discrete energy of motifs. (c) The percentage contribution of the five features $E_{0}, \ldots, E_{4}$. The percentages are calculated from $e_{i}^{2}$ for the first two principal components, where $e_{i}$ is the component corresponding to the $i$ th feature of the first and second principal components.

or long-range Coulomb interactions can induce glassiness in defect-free and dopant-free crystals. The lattice deformation arising from the spin-lattice coupling in sphere packing and long-range Coulomb interaction can similarly lift degeneracy and produce zigzag stripes, rugged freeenergy landscapes, and glassy dynamics. These mechanisms of glassiness in clean crystals are different from those in disordered glasses and cast new light on geometrically frustrated materials, such as charges in organic conductors [20] and spin jamming in magnets [22]. Moreover, the directly observed dynamic heterogeneity and facilitation provide new understanding on the low-temperature spin dynamics in geometrically frustrated materials, which is an important topic in geometrical frustration. We identify 
strong correlations among the static disordered nonstripe spin structure, the static thermodynamic quantity of soft modes [Figs. 1(f)], active spin dynamics (Fig. 5 herein and Fig. S4 of SM [14]), and facilitation regions [Figs. 5 and 6(b)]. In particular, the correlation between soft spots and highly frustrated or high-energy local structures provides a convincing structural signature of soft spots in the context of frustration, and such a correlation is much simpler and clearer than those in amorphous materials [32]. In addition, we obtain consistent quantitative measures of structures and their degrees of facilitation using the supervised SVM in machine learning and unsupervised PCA method (Figs. 8 and 9).

The experiments and simulations emphasize the importance of excitations and facilitated dynamics in spin relaxation at low effective temperatures. We directly observe the increasing hierarchical dynamics as temperature decreases [Figs. 4(d)-4(f)], and experimentally measure excitation lines, the decoupling of two characteristic time scales, $\tau_{x}, \tau_{p}$ in DF, which are responsible for $\mathrm{DH}$ (Fig. 6). Such observations strongly indicate that facilitation theory can be extended to geometrically frustrated systems, where the frustration on lattice serves as the effective constraint in the DF theory. Combining with the analysis of local spin configurations, we further show the correlation between structures and facilitation beyond the DF theory. Although such structural features of excitations support the thermodynamic picture of the glass transition, they are compatible with DF. In fact, DF has been examined in thermodynamic structural glasses in simulation, experiment, and theory. In simulation, the facilitated and hierarchical dynamics have been observed in the Kob-Andersen model [44], which has a disordered critical point [56] and a thermodynamic glass transition [57]. In experiment, colloidal glasses composed of spheres or ellipsoids exhibit both thermodynamic features of glass transition $[58,59]$ and evident facilitations $[47,48]$. In theory, facilitation has also been taken into account within the random-first-order-transition theory [60]. Therefore, our observed facilitated dynamics does not exclude the thermodynamic nature of the glass transition in geometrically frustrated systems. We also note that a recent simulation of buckled hard disks confined in quasi-one-dimension has shown that the system does not exhibit an ideal glass transition and facilitated dynamics is important [36].

Future development of geometrically frustrated colloidal crystals can provide a simple yet versatile platform for studying both glass and geometrical frustration with single-spin dynamics. For example, buckled colloidal spheres in gravity or electric fields can mimic spins in magnetic fields; holographic optical tweezers can organize, manipulate, and perturb multiple spins [9]; printing arrays of microcavities or micromagnets on a substrate are able to assemble colloids onto various geometrically frustrated lattices [11,12]; and doping impurities can induce distinct behaviors in different geometrically frustrated lattices [61].

\section{ACKNOWLEDGMENTS}

We thank Ahmed Alsayed and Arjun Yodh for providing the NIPA colloids, and David Limmer and Weikai Qi for useful discussions. This study was supported by Grants No. RGC-GRF601613, No. RGC-A-HKUST616/14, and No. NSFC11574259 (Y. H.).

\section{APPENDIX A: EXPERIMENTAL METHODS}

The uniform $(<3 \%$ polydispersity) poly $(N$-isopropylacrylamide) (NIPA, NIPAM, or pNIPAM) microgel spheres are little charged with short-range steric repulsions [62] [see Fig. S1(a) of SM [14] ]. Their phase behavior is almost identical to that of hard spheres [63]. Using dynamic light scattering, the hydrodynamic diameter $\sigma$ is measured to have changed linearly from $0.75 \mu \mathrm{m}$ at $24.5^{\circ} \mathrm{C}$ to $0.67 \mu \mathrm{m}$ at $28.5^{\circ} \mathrm{C}$ [Fig. S1(b) of SM [14]]. The hydrodynamic diameter is slightly larger than the effective diameter at $u(r)=1 k_{B} T$ in Fig. S1(a) of SM [14]. The NIPA spheres have almost the same density as the ambient water solution because more than $90 \%$ of the microgel is water.

For the attractive colloid, we add a nonfluorescent liquid dye (D98010 Chromatint jet black 190, Chromatech Incorporated) to induce attraction between PMMA spheres (microparticles $\mathrm{GmbH}$, Berlin) in an aqueous suspension [25]. The mixture of $23 \%$ liquid dye by volume and $77 \%$ water contains $4 \%$ solid dye by weight after drying. The solution is heated at $60^{\circ} \mathrm{C}$ for $5 \mathrm{~h}$ and then sonicated for $1 \mathrm{~min}$. Concentrated dye molecules sometimes aggregate into large clusters, which can induce depletion attraction [25]. This mechanism may cause the observed attraction. The nature of attractive interactions is not well understood. Nevertheless, the tunable attraction can be reproduced in various batches of colloid-dye suspensions; hence, the colloid is robust as a model system [25]. In the sample of dilute PMMA spheres, we use an objective heater to heat the centimeter-sized area in a larger sample at room temperature. The heated area is about $1 \mathrm{~cm}^{2}$ with a uniform temperature. At the edge the this heated area, there is a temperature gradient which pumps dye from the ambient nonheated area to the heated area. By tuning the heating strength, the dye concentration in the region of interest can be well tuned, resulting in tunable attractions, as shown in Fig. S2(a) [14]. Note that typical colloidal interactions such as depletion attraction, fluctuation-induced attraction [64,65], or screened-Coulomb repulsions are all nonpairwise additive at high particle densities in crystal phases; hence, Fig. S2 [14] measures in the dilute suspension only illustrate the tunable attraction qualitatively.

We measure the pair potentials $U(r)$ of the repulsive NIPA spheres [Fig. S1(a) [14]] and attractive PMMA spheres [Fig. S2(a) [14] ] from the equilibrium structures of colloid 
gases [66]. $U(r)$ is calculated from the measured radial distribution function $g(r=|\vec{r}|)=\frac{1}{n^{2}}\left\langle\rho\left(\overrightarrow{r^{\prime}}+\vec{r}, t\right) \rho\left(\overrightarrow{r^{\prime}}, t\right)\right\rangle$ of a dilute monolayer using the Ornstein-Zernike integral equation [66]. Here, $\vec{r}$ is a particle's position, $\rho(\vec{r}, t)=$ $\sum_{j=1}^{N(t)} \delta\left[\vec{r}-\vec{r}_{j}(t)\right], n=\langle\rho\rangle=N / A$ is the area density in the field of view containing $N=\langle N(t)\rangle$ particles, and $\langle\cdots\rangle$ is the average over time. In solving the Ornstein-Zernike equation, the Percus-Yevick approximation is more accurate for hard potentials and the hypernetted-chain approximation is more accurate for soft potentials. Our measured $U(r)$ from the two approximations are consistent, implying that the measurement is reliable.

We place a $1.5-\mu \mathrm{L}$ colloid droplet between a glass slide and a glass cover slip. The droplet spreads between the glass walls under the capillary force and covers most of the $18-\mathrm{mm}^{2}$ area of the coverslip. The glass surfaces are rigorously cleaned before the droplet is placed, to prevent particle attachment. The cover slip and the glass slide are glued together with epoxy so that the wall separation is fixed. The edge of the $18-\mathrm{mm}^{2}$ sample is usually thicker than the central area by less than $2 \sigma$; hence, the $\sim 0.1-\mathrm{mm}^{2}$ field of view has a height difference of only $<0.01 \sigma$ and is sufficiently uniform. We choose an area with an appropriate cell thickness of $H=1.5 \sigma$, where spheres form the buckled monolayer crystal (see the phase diagram of hard spheres confined between hard walls in Ref. [67]). The sample temperature is controlled by an objective heater (Bioptechs). Repulsive spheres can easily selfassemble into crystals at high packing fraction, whereas attractive spheres often form disordered aggregates, small crystallites, or polycrystals with small grain sizes in 2D $[68,69]$. Assembling a large crystalline domain is more challenging in $2 \mathrm{D}$ than in $3 \mathrm{D}$ because $2 \mathrm{D}$ crystals are very soft and subject to more long-wavelength fluctuations. Consequently, we apply a Gaussian beam of light from a $100-\mathrm{W}$ mercury lamp focused by the objective to locally heat a dilute attractive colloidal sample [70]. The black dye absorbs the heat efficiently. The temperature gradient at the edge of the heated area can induce an epitaxial growth of attractive spheres [25]. By gradually increasing the heated area from $400 \mu \mathrm{m}^{2}$ to $0.2 \mathrm{~mm}^{2}$ by adjusting the iris in $10 \mathrm{~h}$, the crystal grows into a millimeter-sized single crystal without grain boundaries. A paraffin film is placed in the optical path to make the temperature uniform in the $0.1-\mathrm{mm}^{2}$ field of view at the center of the millimeter-sized heated area [70]. Repulsive NIPA spheres can directly assemble into buckled crystals without local optical heating and epitaxial growth. The samples are well equilibrated at each temperature step. We record about $45 \mathrm{~min}$ of video at 30 frames/s at each temperature in the repulsive colloidal crystal and at 15 frames/s in different regions of the attractive colloidal crystal using charge-coupled device cameras. The Ising state is identified from the total brightness of each sphere. The threshold of the up and down states is the minimum of the bimodal distribution of the brightness of all particles. Such discretization produces almost exactly $50 \%$ for each Ising state for both attractive and repulsive crystals because gravity is negligible for density-matched NIPA spheres and for PMMA spheres with strong attraction under high packing fractions.

\section{APPENDIX B: SIMULATION METHODS}

Molecular dynamics simulations are performed in the canonical $(N V T)$ ensemble using the LAMMPS simulation package [71]. The system consists of $N=6000$ particles confined between two parallel hard walls with a separation $H=1.5 \sigma$ in a Nose-Hoover thermostat. We use the repulsive Weeks-Chandler-Andersen interparticle pair potential [72]:

$$
U(r)=\left\{\begin{array}{ll}
4 \epsilon\left(\frac{\sigma^{12}}{r^{12}}-\frac{\sigma^{6}}{r^{6}}\right)+\epsilon & r \leq 2^{1 / 6} \sigma \\
0 & r>2^{1 / 6} \sigma
\end{array} .\right.
$$

The length, mass, energy, and time units are set as $\sigma, m, \epsilon$, and $\sqrt{m \sigma^{2} / \epsilon}$, respectively. The temperature is in the unit of $\epsilon / k_{B}$. The time step is set as 0.001 . The particle number density $N /(A H)$ is fixed at 0.7 , where $A$ is the area. The effective diameter of particles $\sigma_{\text {eff }}$ is defined as $U\left(\sigma_{\text {eff }}\right)=1 k_{B} T$, where $\sigma_{\text {eff }}=1.11 \sigma$. Thus, the volume fraction is approximately 0.46 , which falls in the buckled crystalline regime in the hard-sphere phase diagram [67]. The vibrational mode analysis requires no particle swapping and spin flips; hence, we quench the system from a high temperature to a low temperature of $T=0.005$. We obtain different packing configurations using various cooling rates followed by sufficient relaxation $\left(t_{\text {relax }}=10^{4}\right)$. The covariant matrix of displacements is calculated from 60000 -frame trajectories. At each temperature, the results are averaged over 15 independent trials of simulation for enough statistics. The simulation results agree well with the experiments.

Coulomb liquids, also called Coulomb gases, on lattice are a class of models that can describe many problems in 2D statistical mechanics [73], but their glassy dynamics has not been observed until recently [38]. We perform the Monte Carlo simulation of a Coulomb liquid half filling $N=96 \times 96$ sites on a triangular lattice under the periodic boundary condition. This system can be equivalently shifted to global-charge neutral by adding $-q / 2$ charges on every site, i.e., half of the sites filled with $+q / 2$ charges and half of the sites filled with $-q / 2$ charges. Its Hamiltonian is [74]

$$
H=\frac{V}{2} \sum_{i \neq j} \frac{\left(q_{i}-\frac{1}{2}\right)\left(q_{j}-\frac{1}{2}\right)}{\left|\vec{r}_{i}-\vec{r}_{j}\right|},
$$

where the lattice site $i$ at the position $\vec{r}_{i}$ is occupied by charge $q_{i}=1,0$ and $V$ is the interaction energy prefactor. Charge-filled and vacant sites (or $\pm q / 2$ sites) are analogous 
to two Ising states, but the basic move is a charge hopping to its neighboring vacant site rather than a spin flip. We use the Metropolis algorithm with nearest-neighbor updates to mimic real-time dynamics of charge hopping. More details about our Monte Carlo simulation can be found in Ref. [74]. Reference [38] observed a first-order transition between stripes, and the isotropic fluid phase occurred at $T \sim 0.038 \mathrm{~V}$ and glassy dynamics at slightly above $0.038 \mathrm{~V}$. The Coulomb liquid rapidly crystalizes into perfect parallel stripes and can hardly be supercooled at $T<0.038 \mathrm{~V}$. Here, we measure the glassy dynamics at $T=0.04 \mathrm{~V}$ and further observe the stretched exponential behaviors in charge autocorrelation functions and persistence functions of various types of motifs, which have not been reported in previous literature [38].

\section{APPENDIX C: STRUCTURE OF AFIT AND DEFORMABNLE AFIT MODELS}

The degree of frustration in antiferromagnetic Ising spins on an AFIT can be characterized by the average number of frustrated bonds per particle, $\left\langle N_{f}\right\rangle$. At the high $T_{\text {eff }}$ limit, all spins are random; thus, half of the bonds are frustrated and $\left\langle N_{f}\right\rangle=3$. At the ground state, each triangular plaquette has one frustrated bond [Fig. 1(a)]; i.e., one third of the bonds are frustrated and $\left\langle N_{f}\right\rangle=2$. This corresponds to the motif energy $E=\frac{1}{3}\left(6+\frac{6}{2}\right)=3$, because each motif contains six bonds attached to the central spin and six bonds on the edge shared by neighboring motifs with $50 \%$ weight. The ground states of AFIT can be one-to-one mapped to random rhombus tiling in $2 \mathrm{D}$, i.e., cube stacking in $3 \mathrm{D}$ or plane partition in combinatory and number theory $[17,75,76]$. The exact total number of ground states $\Omega$ can be analytically calculated under certain boundary conditions. At the ground state, the boundary effect percolates through the entire system so that it does not have a thermodynamic limit [77]. Different boundary conditions yield $\Omega \sim e^{\alpha N}$ ground states with different values of $\alpha$ [17]. Under the periodic boundary condition, $\alpha=0.323$, and the vast majority of the ground states are disordered, as shown in Fig. 10(a). The ground state of AFIT has an extensive zero-point entropy $S=k_{B} \ln \Omega=\alpha N k_{B}$. This contradicts the third law of thermodynamics, which states that $S \rightarrow 0$ when $T \rightarrow 0$. In real materials, subtle effects at low temperatures, such as lattice deformation and long-range interaction, will relieve degeneracy to satisfy the third law of thermodynamics. These subtle effects give rise to slightly different energies in the degenerated states and result in a rugged energy landscape, thus producing glassy dynamics.

Strictly speaking, a geometrically frustrated material has a finite number of ground states, as required by the third law of thermodynamics. For example, real materials modeled by AFIT must deviate from AFIT due to the subtle effects at low temperatures: its true lowest-energy state at $0 \mathrm{~K}$ is straight parallel stripes; thus, it has three degenerated ground states corresponding to the three equivalent lattice orientations. At finite but very low temperatures, the $2^{\sqrt{N}}$ zigzag stripes have very similar energies to that of straight stripes; hence, the material can be approximately modeled by deformable AFIT, which has $\sim 2^{\sqrt{N}}$ zigzag stripes [see, e.g., Figs. 10(b) and 10(c)] as the ground states and the corresponding ground-state entropy is $\sqrt{N} k_{B} T / N \rightarrow 0$ per particle when $N \rightarrow \infty$, i.e., subextensive. When the temperature is not very low, the subtle effects such as lattice deformation are negligible and the materials can be modeled by AFIT, which has $e^{0.323 N}$ ground states [Fig. 10(a)] with an extensive ground-state entropy $0.323 k_{B} T$ per particle.

In buckled colloidal crystals, the three neighboring spheres tend to form an equilateral triangle in $3 \mathrm{D}$ rather than in $2 \mathrm{D}$ in order to make use of the 1.5 -layer $3 \mathrm{D}$ space more efficiently and have more free volume, especially at high packing fraction. The 3D equilateral triangle projected in $2 \mathrm{D}$ is an isosceles triangle, which can tile the 2D space only by forming zigzag stripe structures composed of motifs 2b and 2c [Fig. 10(b)] (see Ref. [10] for the isosceles triangle tiling). Ground-state zigzag stripes of $N$ spins are fully determined by the $\sqrt{N}$ Ising states at the boundary; hence, $N$ spins have $\sim 2^{\sqrt{N}}$ stripe patterns and subextensive entropy $S \propto \sqrt{N}$ (Fig. 10). We use the fraction of motifs $2 \mathrm{~b}$ and $2 \mathrm{c}, P(2 \mathrm{~b}+2 \mathrm{c})$, to characterize the degree of order. Most of the $e^{0.323 N}$ AFIT ground states have $P(2 \mathrm{~b}+2 \mathrm{c})<1$, and only $e^{\sqrt{N}}$ zigzag stripes have $P(2 \mathrm{~b}+2 \mathrm{c})=1$. They can be viewed as random stacking of ordered 1D chains of alternating up and down spins [Fig. 10(b)], which is analogous to the random stacking of 2D sheets of triangular closest packed spheres in the $z$ direction, i.e., random hexagonal close packing (rhcp) of spheres in 3D. Correspondingly, parallel stripes purely composed of motif 2c are analogous to fcc or hep in 3D. Note that rhcp, hcp, and fcc have an identical closestpacking volume fraction $\phi=74 \%$ and very similar free energies at $\phi<74 \%$ [78]. Similarly, the parallel and zigzag stripes have an identical closest-packing density of isosceles triangles for buckled crystals [10] and very similar free energies below the closest-packing density. Hence, both zigzag stripes and parallel stripes can be viewed as ground states of AFIT on a deformable lattice. Transforming between two zigzag stripe configurations requires a collective flip of at least one chain of $\sqrt{N}$ alternative spins [i.e., one row of spins along the $x$ direction in Fig. 10(b)], which represents a high freeenergy barrier.

Each row of ordered spins in Fig. 10(b) can be stacked in the left and right directions relative to its neighboring row. Hence, the probability that a stripe has a persistence length of $n$ bonds is $P(n)=2^{-n}$. The average persistence length is thus $\sum_{n=1}^{\infty} n P(n)=2$ bonds or, equivalently, 3 spins. The system [Fig. 1(d) herein and Fig. S4(a) of SM [14] ] at high 

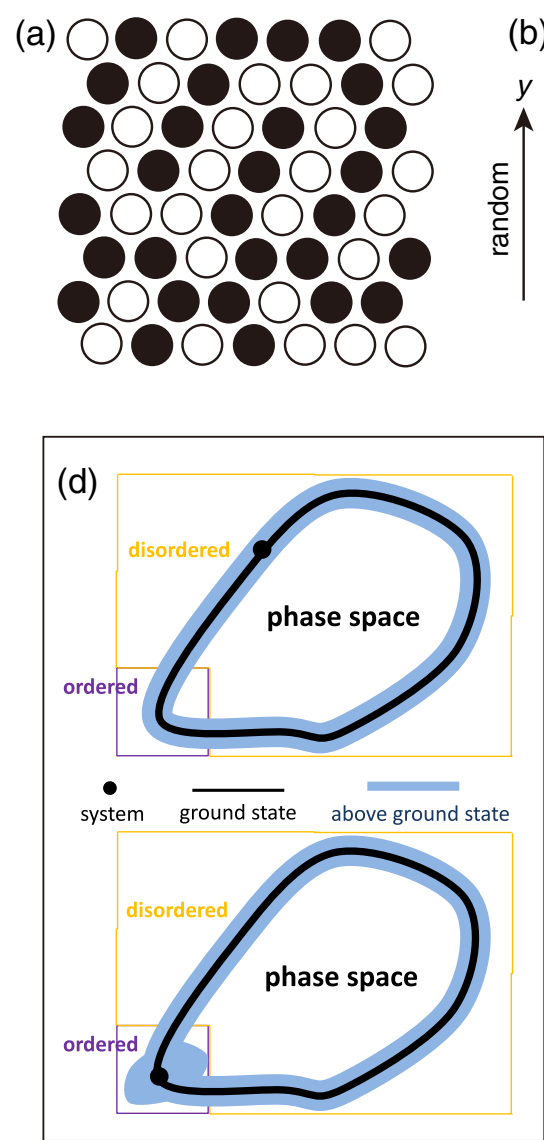
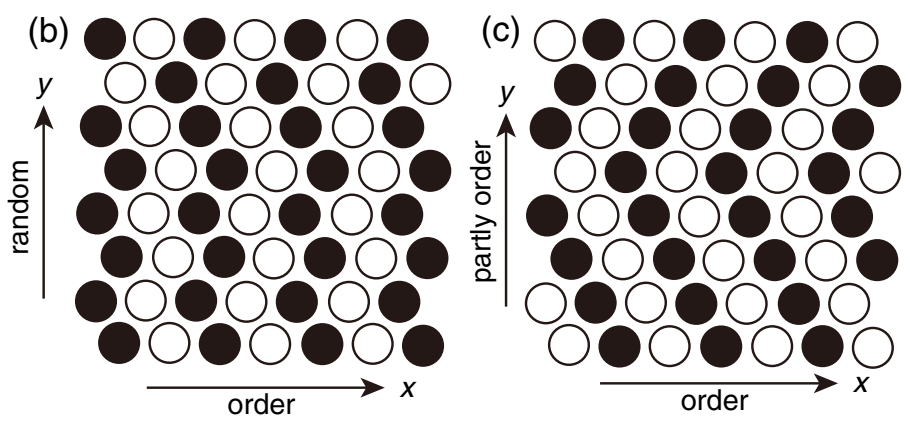

$(e)$

Anisotropic deformation induces global strain

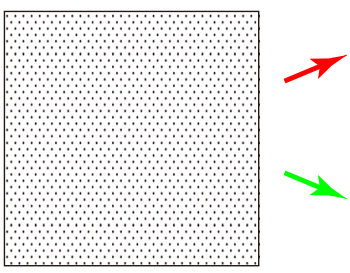

Alternative martensitic deformation induces no global strain

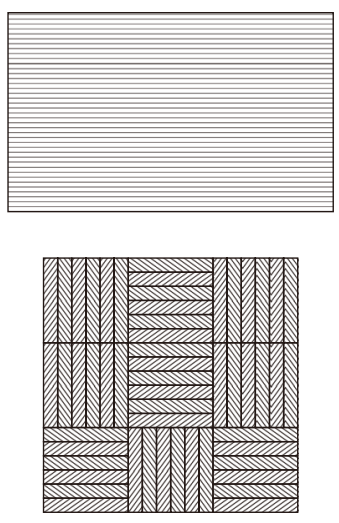

FIG. 10. (a)-(c) Examples of the ground states of AFIT. Panels (b) and (c) are ground states of deformable AFIT, but (a) is not. (a) Among $e^{0.323 N}$ ground states, a typical one has disorder structures composed of motifs $0,1,2 \mathrm{~b}, 2 \mathrm{c}$, and $3 \mathrm{c}$. (b) Random zigzag stripe patterns can be viewed as ordered 1D arrays with alternative Ising states along the $x$ direction stacking randomly in the $y$ direction; hence, there are $2^{\sqrt{N}}$ ways of stacking, i.e., $2^{\sqrt{N}}=e^{\ln 2 \sqrt{N}} \sim e^{\sqrt{N}}$ patterns. The factor $\ln 2$ depends on the boundary condition, and thus is neglected in the main text. To jump to another ground state of the deformation AFIT model, one row of spin ( $\sqrt{N}$ spins) needs to shift simultaneously, which corresponds to a high-energy barrier and results in glassiness. Similarly, in buckled crystals, when the effective temperature is lowered towards the ground state, there are less free spins (motif $3 \mathrm{c}$ ) and, consequently, jumping from one low-temperature state to the other requires more spins to collectively flip; thus, it is more glassy. (c) More ordered zigzag stripes with longer persistence lengths due to the order-bydisorder effect illustrated in (d). (d) The order-by-disorder effect illustrated in phase space. We replot Fig. 3 of Ref. [5]. Ordered real-space structures correspond to the bottom left region in the phase space. In the top panel without the order-by-disorder effect, the system tends to be in a disordered state because the vast majority of ground states are disordered. The bottom panel depicts a phase space with the order-bydisorder effect: the system tends to be in an ordered state because thermal fluctuation can explore more phase space in the ordered region slightly above the ground state. As quenching towards the ground state (i.e., from the blue region to the black region), the system tends to inherit its ordered structure in a rugged free-energy landscape. (e) Schematic of two types of deformation in a solid-solid transition. When a crystalline domain of the parent phase transforms to a single crystalline domain of the product phase, the change in its shape induces a high strain energy. In real materials, the parent crystal usually transforms into small martensitic domains with alternative lattice orientations to cancel the strain energy. This mechanism is likely responsible for the formation of the medium-sized rather than the large-sized stripe domains in Fig. 1(d) herein and Fig. S4(a) of SM [14].

$\phi$ near the ground state, however, contain more ordered stripes with a typical persistence length of 5 spheres. We attribute this to the "order-by-disorder" effect typically encountered in geometrically frustrated systems: although there are dominant numbers of disordered strip configurations at the exact ground state, the system slightly above the ground state will stay at a more ordered state because ordered structures have higher entropies (more free volume for colloids) [Fig. 10(d)]. On the other hand, forming a large domain of parallel (i.e., nonzigzag) stripes by increasing $\phi$ would cause a large-scale shape deformation associated with a large strain energy [Fig. 10(e)]. Hence, the persistence lengths of stripes cannot be too long, and stripes form medium-sized domains with alternative orientations at high $\phi$ in Fig. 1(d) herein and Fig. S4(a) of SM [14]. The alternative stripe orientations can cancel out the stress, analogous to martensitic structures in polycrystals, as illustrated in Fig. 10(e). 
[1] R. Moessner and A. P. Ramirez, Geometrical Frustration, Phys. Today 59, 24 (2006).

[2] G. Wannier, Antiferromagnetism. The Triangular Ising Net, Phys. Rev. 79, 357 (1950).

[3] C. Castelnovo, R. Moessner, and S. L. Sondhi, Magnetic Monopoles in Spin Ice, Nature (London) 451, 42 (2008).

[4] S. Bramwell, S. Giblin, S. Calder, R. Aldus, D. Prabhakaran, and T. Fennell, Measurement of the Charge and Current of Magnetic Monopoles in Spin Ice, Nature (London) 461, 956 (2009).

[5] R. Moessner, Magnets with Strong Geometric Frustration, Can. J. Phys. 79, 1283 (2001).

[6] R. Wang, C. Nisoli, R. Freitas, J. Li, W. McConville, B. Cooley, M. Lund, N. Samarth, C. Leighton, V. Crespi et al., Artificial Spin Ice' in a Geometrically Frustrated Lattice of Nanoscale Ferromagnetic Islands, Nature (London) 439, 303 (2006).

[7] C. Nisoli, R. Moessner, and P. Schiffer, Colloquium: Artificial Spin Ice: Designing and Imaging Magnetic Frustration, Rev. Mod. Phys. 85, 1473 (2013).

[8] H. Hilgenkamp, H.-J. H. Smilde, D. H. Blank, G. Rijnders, H. Rogalla, J. R. Kirtley, C. C. Tsuei et al., Ordering and Manipulation of the Magnetic Moments in Large-Scale Superconducting $\pi$-Loop Arrays, Nature (London) 422 , 50 (2003).

[9] A. Libal, C. Reichhardt, and C. J. Olson Reichhardt, Realizing Colloidal Artificial Ice on Arrays of Optical Traps, Phys. Rev. Lett. 97, 228302 (2006).

[10] Y. Han, Y. Shokef, A. M. Alsayed, P. Yunker, T. C. Lubensky, and A. G. Yodh, Geometric Frustration in Buckled Colloidal Monolayers, Nature (London) 456, 898 (2008).

[11] P. Tierno, Geometric Frustration of Colloidal Dimers on a Honeycomb Magnetic Lattice, Phys. Rev. Lett. 116, 038303 (2016).

[12] A. Ortiz-Ambriz and P. Tierno, Engineering of Frustration in Colloidal Artificial Ices Realized on Microfeatured Grooved Lattices, Nat. Commun. 7, 10575 (2016).

[13] L. Berthier and G. Biroli, Theoretical Perspective on the Glass Transition and Amorphous Materials, Rev. Mod. Phys. 83, 587 (2011).

[14] See Supplemental Material at http://link.aps.org/ supplemental/10.1103/PhysRevX.7.021030 for the details about experiment, analysis and movies.

[15] K. Chen, T. Still, S. Schoenholz, K. B. Aptowicz, M. Schindler, A. C. Maggs, A. J. Liu, and A. G. Yodh, Phonons in Two-Dimensional Soft Colloidal Crystals, Phys. Rev. E 88, 022315 (2013).

[16] L. Balents, Spin Liquids in Frustrated Magnets, Nature (London) 464, 199 (2010).

[17] Y. Han, Phase-Space Networks of Geometrically Frustrated Systems, Phys. Rev. E 80, 051102 (2009).

[18] J.-R. Lee and S. Teitel, Dense Two-Dimensional Classical Coulomb Gas on a Triangular Lattice, Phys. Rev. Lett. 66, 2100 (1991).

[19] I. Klich, S.-H. Lee, and K. Iida, Glassiness and Exotic Entropy Scaling Induced by Quantum Fluctuations in a Disorder-Free Frustrated Magnet, Nat. Commun. 5, 3497 (2014).
[20] F. Kagawa, T. Sato, K. Miyagawa, K. Kanoda, Y. Tokura, K. Kobayashi, R. Kumai, and Y. Murakami, ChargeCluster Glass in an Organic Conductor, Nat. Phys. 9, 419 (2013).

[21] M. J. P. Gingras, C. V. Stager, N. P. Raju, B. D. Gaulin, and J. E. Greedan, Static Critical Behavior of the Spin-Freezing Transition in the Geometrically Frustrated Pyrochlore Antiferromagnet $\mathrm{Y}_{2} \mathrm{Mo}_{2} \mathrm{O}_{7}$, Phys. Rev. Lett. 78, 947 (1997).

[22] J. Yang, A. Samarakoon, S. Dissanayake, H. Ueda, I. Klich, K. Iida, D. Pajerowski, N. P. Butch, Q. Huang, J. R. Copley et al., Spin Jam Induced by Quantum Fluctuations in a Frustrated Magnet, Proc. Natl. Acad. Sci. U.S.A. 112, 11519 (2015).

[23] C. P. Royall and S. R. Williams, The Role of Local Structure in Dynamical Arrest, Phys. Rep. 560, 1 (2015).

[24] A. M. Alsayed, M. F. Islam, J. Zhang, P. J. Collings, and A. G. Yodh, Premelting at Defects within Bulk Colloidal Crystals, Science 309, 1207 (2005).

[25] B. Li, F. Wang, D. Zhou, Y. Peng, R. Ni, and Y. Han, Modes of Surface Premelting in Colloidal Crystals Composed of Attractive Particles, Nature (London) 531, 485 (2016).

[26] J. C. Crocker and D. G. Grier, Methods of Digital Video Microscopy for Colloidal Studies, J. Colloid Interface Sci. 179, 298 (1996).

[27] G. Wannier, Antiferromagnetism. The Triangular Ising Net, Phys. Rev. B 7, 5017 (1973).

[28] E. Ma, Tuning Order in Disorder, Nat. Mater. 14, 547 (2015).

[29] T. Kawasaki, T. Araki, and H. Tanaka, Correlation between Dynamic Heterogeneity and Medium-Range Order in TwoDimensional Glass-Forming Liquids, Phys. Rev. Lett. 99, 215701 (2007).

[30] D. Kaya, N. Green, C. Maloney, and M. Islam, Normal Modes and Density of States of Disordered Colloidal Solids, Science 329, 656 (2010).

[31] K. Chen, M. L. Manning, P. J. Yunker, W. G. Ellenbroek, Z. Zhang, A. J. Liu, and A. G. Yodh, Measurement of Correlations between Low-Frequency Vibrational Modes and Particle Rearrangements in Quasi-Two-Dimensional Colloidal Glasses, Phys. Rev. Lett. 107, 108301 (2011).

[32] M. L. Manning and A. J. Liu, Vibrational Modes Identify Soft Spots in a Sheared Disordered Packing, Phys. Rev. Lett. 107, 108302 (2011).

[33] J. Ding, S. Patinet, M. L. Falk, Y. Cheng, and E. Ma, Soft Spots and Their Structural Signature in a Metallic Glass, Proc. Natl. Acad. Sci. U.S.A. 111, 14052 (2014).

[34] H. Tanaka, T. Kawasaki, H. Shintani, and K. Watanabe, Critical-like Behaviour of Glass-Forming Liquids, Nat. Mater. 9, 324 (2010).

[35] Y. Shokef, A. Souslov, and T. C. Lubensky, Order by Disorder in the Antiferromagnetic Ising Model on an Elastic Triangular Lattice, Proc. Natl. Acad. Sci. U.S.A. 108, 11804 (2011).

[36] M. Z. Yamchi, S. S. Ashwin, and R. K. Bowles, FragileStrong Fluid Crossover and Universal Relaxation Times in a Confined Hard-Disk Fluid, Phys. Rev. Lett. 109, 225701 (2012).

[37] J. Phillips, Stretched Exponential Relaxation in Molecular and Electronic Glasses, Rep. Prog. Phys. 59, 1133 (1996). 
[38] S. Mahmoudian, L. Rademaker, A. Ralko, S. Fratini, and V. Dobrosavljević, Glassy Dynamics in Geometrically Frustrated Coulomb Liquids without Disorder, Phys. Rev. Lett. 115, 025701 (2015).

[39] T. Sato, F. Kagawa, K. Kobayashi, K. Miyagawa, K. Kanoda, R. Kumai, Y. Murakami, and Y. Tokura, Emergence of Nonequilibrium Charge Dynamics in a ChargeCluster Glass, Phys. Rev. B 89, 121102 (2014).

[40] R. L. Jack, L. Berthier, and J. P. Garrahan, Static and Dynamic Length Scales in a Simple Glassy Plaquette Model, Phys. Rev. E 72, 016103 (2005).

[41] E. R. Weeks, J. C. Crocker, A. C. Levitt, A. Schofield, and D. A. Weitz, Three-Dimensional Direct Imaging of Structural Relaxation Near the Colloidal Glass Transition, Science 287, 627 (2000).

[42] C. Donati, S. C. Glotzer, P. H. Poole, W. Kob, and S. J. Plimpton, Spatial Correlations of Mobility and Immobility in a Glass-Forming Lennard-Jones Liquid, Phys. Rev. E 60, 3107 (1999).

[43] Z. Zheng, F. Wang, Y. Han et al., Glass Transitions in Quasi-Two-Dimensional Suspensions of Colloidal Ellipsoids, Phys. Rev. Lett. 107, 065702 (2011).

[44] A. S. Keys, L. O. Hedges, J. P. Garrahan, S. C. Glotzer, and D. Chandler, Excitations Are Localized and Relaxation Is Hierarchical in Glass-Forming Liquids, Phys. Rev. X 1, 021013 (2011).

[45] D. Chandler and J. Garrahan, Dynamics on the Way to Forming Glass: Bubbles in Space-Time, Annu. Rev. Phys. Chem. 61, 191 (2010).

[46] J. P. Garrahan, P. Sollich, and C. Toninelli, Dynamical Heterogeneities in Glasses, Colloids and Granular Media and Jamming Transitions, International Series of Monographs in Physics (Oxford University Press, Oxford, England, 2011), Chap 10, pp. 341-369.

[47] C. K. Mishra, K. H. Nagamanasa, R. Ganapathy, A. Sood, and S. Gokhale, Dynamical Facilitation Governs Glassy Dynamics in Suspensions of Colloidal Ellipsoids, Proc. Natl. Acad. Sci. U.S.A. 111, 15362 (2014).

[48] S. Gokhale, K. H. Nagamanasa, R. Ganapathy, and A. Sood, Growing Dynamical Facilitation on Approaching the Random Pinning Colloidal Glass Transition, Nat. Commun. 5, 4685 (2014).

[49] S. Gokhale, A. Sood, and R. Ganapathy, Deconstructing the Glass Transition through Critical Experiments on Colloids, Adv. Phys. 65, 363 (2016).

[50] L. O. Hedges, L. Maibaum, D. Chandler, and J. P. Garrahan, Decoupling of Exchange and Persistence Times in Atomistic Models of Glass Formers, J. Chem. Phys. 127, 211101 (2007).

[51] M. Vogel and S.C. Glotzer, Spatially Heterogeneous Dynamics and Dynamic Facilitation in a Model of Viscous Silica, Phys. Rev. Lett. 92, 255901 (2004).

[52] Y. S. Elmatad and A. S. Keys, Manifestations of Dynamical Facilitation in Glassy Materials, Phys. Rev. E 85, 061502 (2012).

[53] S. S. Schoenholz, E. D. Cubuk, D. M. Sussman, E. Kaxiras, and A.J. Liu, A Structural Approach to Relaxation in Glassy Liquids, Nat. Phys. 12, 469 (2016).

[54] C.-C. Chang and C.-J. Lin, LIBSVM: A Library for Support Vector Machines, ACM Trans. Intell. Syst. Technol. 2, 1 (2011).
[55] G. James, D. Witten, T. Hastie, and R. Tibshirani, An Introduction to Statistical Learning (Springer, New York, 2013), Vol. 6.

[56] L. Berthier and R. L. Jack, Evidence for a Disordered Critical Point in a Glass-Forming Liquid, Phys. Rev. Lett. 114, 205701 (2015).

[57] M. Ozawa, W. Kob, A. Ikeda, and K. Miyazaki, Equilibrium Phase Diagram of a Randomly Pinned Glass-Former, Proc. Natl. Acad. Sci. U.S.A. 112, 6914 (2015).

[58] Z. Zheng, R. Ni, F. Wang, M. Dijkstra, Y. Wang, and Y. Han, Structural Signatures of Dynamic Heterogeneities in Monolayers of Colloidal Ellipsoids, Nat. Commun. 5, 3829 (2014).

[59] K. H. Nagamanasa, S. Gokhale, A. Sood, and R. Ganapathy, Direct Measurements of Growing Amorphous Order and Non-Monotonic Dynamic Correlations in a Colloidal Glass-Former, Nat. Phys. 11, 403 (2015).

[60] S. M. Bhattacharyya, B. Bagchi, and P. G. Wolynes, Facilitation, Complexity Growth, Mode Coupling, and Activated Dynamics in Supercooled Liquids, Proc. Natl. Acad. Sci. U.S.A. 105, 16077 (2008).

[61] A. Libál, C. O. Reichhardt, and C. Reichhardt, Doped Colloidal Artificial Spin Ice, New J. Phys. 17, 103010 (2015).

[62] Y. Peng, Z.-R. Wang, A. M. Alsayed, A. G. Yodh, Y. Han, Melting of Multilayer Colloidal Crystals Confined between Two Walls, Phys. Rev. E 83, 011404 (2011).

[63] A. M. Alsayed, M. F. Islam, J. Zhang, P. J. Collings, and A. G. Yodh, Premelting at Defects within Bulk Colloidal Crystals, Science 309, 1207 (2005).

[64] C. Hertlein, L. Helden, A. Gambassi, S. Dietrich, and C. Bechinger, Direct Measurement of Critical Casimir Forces, Nature (London) 451, 172 (2008).

[65] J. R. Edison, N. Tasios, S. Belli, R. Evans, R. van Roij, and M. Dijkstra, Critical Casimir Forces and Colloidal Phase Transitions in a Near-Critical Solvent: A Simple Model Reveals a Rich Phase Diagram, Phys. Rev. Lett. 114, 038301 (2015).

[66] S. H. Behrens and D. G. Grier, Pair Interaction of Charged Colloidal Spheres Near a Charged Wall, Phys. Rev. E 64, 050401 (2001).

[67] A. Fortini and M. Dijkstra, Phase Behaviour of Hard Spheres Confined Between Parallel Hard Plates: Manipulation of Colloidal Crystal Structures by Confinement, J. Phys. Condens. Matter 18, L371 (2006).

[68] W. M. Jacobs, A. Reinhardt, and D. Frenkel, Rational Design of Self-Assembly Pathways for Complex Multicomponent Structures, Proc. Natl. Acad. Sci. U.S.A. 112, 6313 (2015).

[69] B. Li, D. Zhou, and Y. Han, Assembly and Phase Transitions of Colloidal Crystals, Nat. Rev. Mater. 1, 15011 (2016).

[70] Z. Wang, F. Wang, Y. Peng, Z. Zheng, and Y. Han, Imaging the Homogeneous Nucleation During the Melting of Superheated Colloidal Crystals, Science 338, 87 (2012).

[71] S. Plimpton, Fast Parallel Algorithms for Short-Range Molecular Dynamics, J. Comput. Phys. 117, 1 (1995).

[72] J. D. Weeks, D. Chandler, and H. C. Andersen, Role of Repulsive Forces in Determining the Equilibrium Structure of Simple Liquids, J. Chem. Phys. 54, 5237 (1971). 
[73] B. Nienhuis, Critical Behavior of Two-Dimensional Spin Models and Charge Asymmetry in the Coulomb Gas, J. Stat. Phys. 34, 731 (1984).

[74] A. B. Kolton, D. R. Grempel, and D. Domínguez, Heterogeneous Dynamics of the Three-Dimensional Coulomb Glass Out of Equilibrium, Phys. Rev. B 71, 024206 (2005).

[75] H. W. J. Blöte and B. Nienhuis, Fully Packed Loop Model on the Honeycomb Lattice, Phys. Rev. Lett. 72, 1372 (1994).
[76] G. E. Andrews and K. Eriksson, Integer Partitions (Cambridge University Press, Cambridge, England, 2004).

[77] N. Destainville, Entropy and Boundary Conditions in Random Rhombus Tilings, J. Phys. A 31, 6123 (1998).

[78] P. G. Bolhuis, D. Frenkel, S.-C. Mau, and D. A. Huse, Entropy Difference between Crystal Phases, Nature (London) 388, 235 (1997). 